\title{
EXISTENCE AND UNIQUENESS TO THE CAUCHY PROBLEM FOR LINEAR AND SEMILINEAR PARABOLIC EQUATIONS WITH LOCAL CONDITIONS.*
}

\author{
Gerardo Rubio ${ }^{1}$
}

\begin{abstract}
We consider the Cauchy problem in $\mathbb{R}^{d}$ for a class of semilinear parabolic partial differential equations that arises in some stochastic control problems. We assume that the coefficients are unbounded and locally Lipschitz, not necessarily differentiable, with continuous data and local uniform ellipticity. We construct a classical solution by approximation with linear parabolic equations. The linear equations involved can not be solved with the traditional results. Therefore, we construct a classical solution to the linear Cauchy problem under the same hypotheses on the coefficients for the semilinear equation. Our approach is using stochastic differential equations and parabolic differential equations in bounded domains.

Finally, we apply the results to a stochastic optimal consumption problem.
\end{abstract}

Résumé. Nous considérons le problème de Cauchy dans $\mathbb{R}^{d}$ pour une classe d'équations aux dérivées partielles paraboliques semi linéaires qui se pose dans certains problèmes de contrôle stochastique. Nous supposons que les coefficients ne sont pas bornés et sont localement Lipschitziennes, pas nécessairement différentiables, avec des données continues et ellipticité local uniforme. Nous construisons une solution classique par approximation avec les équations paraboliques linéaires. Les équations linéaires impliquées ne peuvent être résolues avec les résultats traditionnels. Par conséquent, nous construisons une solution classique au problème de Cauchy linéaire sous les mêmes hypothèses sur les coefficients pour l'équation semi-linéaire. Notre approche utilise les équations différentielles stochastiques et les équations différentielles paraboliques dans les domaines bornés.

Enfin, nous appliquons les résultats à un problème stochastique de consommation optimale.

\section{INTRODUCTION}

In the Theory of Stochastic Control, one of the techniques for studying the value function is the Dynamic Programming Principle and the Hamilton-Jacobi-Bellman equations (HJB equations). Generally, HJB equations are nonlinear partial differential equations. In many interesting problems (see e.g. [42], [37], [19], [9], [28], [29] and [39]) the HJB equation can be reduced to an equation of the form

$$
\begin{aligned}
-u_{t}(t, x)+\sum_{i j} a_{i j}(t, x) D_{i j}(t, x)+\sup _{\alpha \in \Lambda}\left\{\mathcal{L}^{\alpha}[u](t, x)\right\} & =0, \quad \text { in }(0, \infty) \times \mathbb{R}^{d}, \\
u(0, x) & =h(x), \quad x \in \mathbb{R}^{d},
\end{aligned}
$$

\section{Article published online by EDP Sciences and available at http://www.esaim-proc.org or http://dx.doi.org/}

* This work was partially supported by grant PAPIIT-DGAPA-UNAM IN103660, IN117109 and CONACYT 180312, México.

${ }^{1}$ Departamento de Matemáticas, Facultad de Ciencias, UNAM, MÉXICO

e-mail: grubioh@yahoo.com 
where $\left\{a_{i j}\right\}=a=\sigma \sigma^{\prime}$ and

$$
\mathcal{L}^{\alpha}[u](t, x):=\sum_{i} b_{i}(t, x, \alpha) D_{i} u(t, x)+c(t, x, \alpha) u(t, x)+f(t, x, \alpha) .
$$

In this paper we study the existence and uniqueness of a classical solution to equation (1) when the coefficients $\sigma, b, c$ and $f$ are locally Hölder in $t$ and locally Lipschitz in $(x, \alpha)$, not necessarily differentiable, $\sigma$ and $b$ have linear growth, $c$ is bounded from above and $f$ has a polynomial growth of any order. $h$ is a continuous function with polynomial growth and $\Lambda \subset \mathbb{R}^{m}$ is a connected compact set. We assume the ellipticity condition to be local, that is, for any $[0, T] \times A \subset[0, \infty) \times \mathbb{R}^{d}$ there exists $\lambda(T, A)$ such that $\sum a_{i j}(t, x) \xi_{i} \xi_{j} \geq \lambda(T, A)\|\xi\|^{2}$ for all $x, \xi \in A$ and $t \in[0, T]$.

We construct a solution by approximation with linear parabolic equations. Despite the approximation technique is standard (see [21], Appendix E), the linear equations involved can not be solved with the traditional results. Therefore, we study the existence of a classical solution to the Cauchy problem for a second order linear parabolic equation. Let $\mathcal{L}$ be the differential operator

$$
\mathcal{L}[u](t, x)=\sum_{i, j} a_{i j}(t, x) D_{i j} u(t, x)+\sum_{i} b_{i}(t, x) D_{i} u(t, x) .
$$

Then the Cauchy problem is

$$
\begin{aligned}
-u_{t}(t, x)+\mathcal{L}[u](t, x)+c(t, x) u(t, x) & =-f(t, x) \quad(t, x) \in(0, \infty) \times \mathbb{R}^{d}, \\
u(0, x) & =h(x) \quad \text { for } x \in \mathbb{R}^{d} .
\end{aligned}
$$

We prove the existence and uniqueness of a classical solution to equation (2) when the coefficients fulfil the same hypotheses of the ones of the semilinear problem (1). Actually, for the linear problem we allow the quadratic form to degenerate in a closed, connected set $\Sigma \subset \mathbb{R}^{d}$, that is, for all $A \subset \mathbb{R}^{d} \backslash \Sigma$ and $T>0$, there exists $\lambda(T, A)>0$ such that for all $(t, x) \in[0, T] \times \bar{A}$ and $\xi \in \mathbb{R}^{d}$

$$
\sum_{i, j} a_{i j}(t, x) \xi_{i} \xi_{j} \geq \lambda(T, A)\|\xi\|^{2},
$$

and for all $(t, x) \in[0, T] \times \partial \Sigma$

$$
\begin{gathered}
\sum_{i, j} a_{i j}(t, x) \nu_{i} \nu_{j}=0 \\
\sum_{i} b_{i}(t, x) D_{i} \rho(x)+\sum_{i, j} a_{i, j}(t, x) D_{i j} \rho(x) \geq 0
\end{gathered}
$$

where $\nu$ represents the inward normal and $\rho(x)=\operatorname{dist}(x, \partial \Sigma)$. The linear problem is then solved in the set $(0, \infty) \times \mathbb{R}^{d} \backslash \Sigma$.

Finally, we study the value function for a stochastic consumption model that maximizes the utility function over all consumption strategies. In this case, the HJB equation is a semilinear parabolic equation of the form of equation (1). To prove that the value function is the solution to the HJB equation, we prove both, a Verification Theorem and an Existence one. The Verification Theorem asserts that if a classical solution to the HJB equation exists, then it has to be the value function. This Theorem also proves the existence of an optimal consumption strategy. For the Existence, we follow the same lines used in the proof for equation (1). However, this problem is degenerated on the set $\{x=0\}$ and so we apply the result proved for the linear parabolic equations.

For the linear parabolic problem, the existence of a fundamental solution to the Cauchy problem via the parametrix method, is well known in the case of bounded Hölder coefficients of $\mathcal{L}$ (see [24] for detailed description of this theory). 
Linear parabolic equations with unbounded coefficients have been studied in great detail in the last sixty years. The existence, uniqueness and regularity of the solution to the Cauchy problem has been studied under a wide range of assumptions on the coefficients. See [8], [2], [7] and [17] for a classical approach with fundamental solutions; in [16], [36], [35], [15] and [6] the problem is studied using the Theory of Semigroups (see [34] for a survey of these ideas); see also [10], [11], [12] and [13] for a probabilistic approach.

Our method is using stochastic differential equations and parabolic differential equations in bounded domains. First, we propose as a solution to equation (2), a functional of the solution to a SDE

$$
v(t, x)=\mathbb{E}_{x}\left[\int_{0}^{t} e^{\int_{0}^{s} c(t-r, X(r)) d r} f(t-s, X(s)) d s+e^{\int_{0}^{t} c(t-r, X(r)) d r} h(X(t))\right]
$$

where

$$
d X(s)=b(t-s, X(s)) d s+\sigma(t-s, X(s)) d W(s) .
$$

Using the continuity of the paths we prove that this function is continuous in $[0, \infty) \times \mathbb{R}^{d}$. With the theory of parabolic equations in bounded domains, we prove that $v$ is $C^{1,2}$ locally and finally we prove that it solves the Cauchy problem. This kind of idea has been used for several partial differential problems (see [23], [3] and [18]). In the book by Krylov [32], a similar result is given with the extra assumptions that all the functions, $a_{i j}, b_{i}$, $c, f$ and $h$ are twice continuously differentiable in $x$. In that case, it was proved that the flow of the SDE is differentiable and so the function $v \in C^{1,2}$ and solves the Cauchy problem. It is important to note that with our assumptions the flow may not be differentiable.

In recent works (see [35], [22], [4], [6], [5], [30], among others), broader assumptions about the growth of the coefficients have been made. In these papers the authors assume the existence of a function $\varphi \in C^{1,2}\left((0, T) \times \mathbb{R}^{d}\right)$ such that

and for some $\lambda>0$

$$
\lim _{|x| \rightarrow \infty} \inf _{0 \leq t \leq T} \varphi(t, x)=\infty
$$

$$
\sup _{[0, T] \times \mathbb{R}^{d}}\left\{\left(-\frac{\partial}{\partial t}+\mathcal{L}\right) \varphi(t, x)-\lambda \varphi(t, x)\right\}<\infty .
$$

This is a generalization on the hypotheses made in this paper on the coefficients $b$ and $\sigma($ the function $\varphi(t, x)=$ $\|x\|^{2}$ satisfies both conditions). The ideas in our paper may be repeated under this broader assumption. However, the existence of moments for the stochastic process $X(s)$ associated to the semigroup generated by $\mathcal{L}$ is not clear and so we should work with bounded data $\left(f, h \in C_{b}\right)$ to guarantee the existence of the proposed solution $v$. Because many interesting stochastic control problems require the non-boundedness of the data, we work with processes for which the growth of the moments can be controlled. See Remark 2.3 at the end of section 2.3 for a more detailed discussion about this hypothesis.

This paper is divided as follows: In section 1 we present the notation used throughout this work. Section 2 is dedicated to the study of the linear parabolic problem (2). In subsection 2.1 we introduce the notation and the hypotheses used throughout this part. Subsection 2.2 presents the main result for the linear parabolic differential equation. In this section we prove that if the function $v$ is smooth, then it has to be the solution to the Cauchy problem. Subsection 2.3 is devoted to prove the required differentiability for the candidate function. The third part is dedicated to the semilinear problem (1) and it is contained in section 3 . In section 4 we present the optimal consumption problem. Finally, in section 5 the reader will find some of the results used in the proofs of this work.

\section{Notation.}

In this section we present the notation used in this work.

Let $x \in \mathbb{R}^{d}$ and $A \in \mathcal{M}\left(\mathbb{R}^{d} \times \mathbb{R}^{d}\right)$. We denote by $\|x\|$ the usual norm in $\mathbb{R}^{d}$. For the matrix norm we consider $\|A\|^{2}:=\operatorname{tr} A A^{\prime}=\sum_{i, j} A_{i j}^{2}$. 
If $\mu$ is a locally Lipschitz function defined in some set $D$, then for any bounded open set $A$ for which $\bar{A} \subset D$, we denote by $K_{\mu}(A)$ and $L_{\mu}(A)$, constants such that

$$
\begin{gathered}
K_{\mu}(A):=\sup _{x \in A}\|\mu(x)\|<\infty, \\
L_{\mu}(A):=\sup _{x, y \in A, x \neq y} \frac{\|\mu(x)-\mu(y)\|}{\|x-y\|}<\infty .
\end{gathered}
$$

If $\nu:[0, \infty) \rightarrow \mathbb{R}^{d}$, then for all $T>0$

$$
\|\nu\|_{T}:=\sup _{0 \leq s \leq T}\|\nu(s)\| .
$$

The space $C_{\text {loc }}^{1,2, \beta}\left((0, \infty) \times \mathbb{R}^{d}\right)$ is the space of all functions such that they and all their derivatives up to the second order in $x$ and first order in $t$, are locally Hölder of order $\beta$.

We denote by $H L^{k, m, \beta_{0}, \beta_{1}}((0, \infty) \times D) \subset C^{k, m}((0, \infty) \times D)$, with $\beta_{0}, \beta_{1} \in(0,1]$, the space of all continuous function such that all their derivatives up to order $k$ in $t$ and order $m$ in $x$, are locally Hölder continuous of order $\beta_{0}$ in $t$ and locally Hölder continuous of order $\beta_{1}$ in $x$. If $\beta_{1}=1$ we denote by $H L^{k, m, \beta_{0}}((0, \infty) \times D)$.

We use the following notation for the Sobolev and Hölder norms. Let $R \subset[0, \infty) \times \mathbb{R}^{d}, f: R \rightarrow \mathbb{R}$ be an arbitrary function, $\alpha \in(0,1]$ and $1<p<\infty$, then

$$
\begin{aligned}
\|f\|_{R}:= & \sup _{(t, z) \in R}|f(t, z)|, \\
|f|_{R}^{\alpha}:= & \|f\|_{R}+\sup _{\left(t, z_{1}\right) \neq\left(t, z_{2}\right) \in R} \frac{\left|f\left(t, z_{1}\right)-f\left(t, z_{2}\right)\right|}{\left|z_{1}-z_{2}\right|^{\alpha}} \\
& +\sup _{\left(t_{1}, z\right) \neq\left(t_{2}, z\right) \in R} \frac{\left|f\left(t_{1}, z\right)-f\left(t_{2}, z\right)\right|}{\left|t_{1}-t_{2}\right|^{\alpha / 2}}, \\
|f|_{R}^{1, \alpha}:= & |f|_{R}^{\alpha}+\sum_{i}\left|D_{i} f\right|_{R}^{\alpha}, \\
|f|_{R}^{2, \alpha}:= & |f|_{R}^{1, \alpha}+\left|f_{t}\right|_{R}^{\alpha}+\sum_{i, j}\left|D_{i j} f\right|_{R}^{\alpha}, \\
\|f\|_{p ; R}:= & \left(\int_{R}|f|^{p} d z\right)^{\frac{1}{p}}, \\
\|f\|_{2, p ; R}:= & \|f\|_{p ; R}+\left\|f_{t}\right\|_{p ; R}+\sum_{i}\left\|D_{i} f\right\|_{p ; R}+\sum_{i, j}\left\|D_{i j} f\right\|_{p ; R} .
\end{aligned}
$$

\section{LINEAR PARABOLIC EQUATIONS.}

\subsection{Preliminaries and hypotheses.}

In this section we present the hypotheses and some notation used in this work.

\subsubsection{Stochastic differential equation.}

Let $\left(\Omega, \mathcal{F}, \mathbb{P},\left\{\mathcal{F}_{s}\right\}_{s \geq 0}\right)$ be a complete filtered probability space and let $\{W\}=\left\{W_{i}\right\}_{i=1}^{d}$ be a $d$-dimensional Brownian motion defined in it. Consider the following stochastic differential equation

$$
d X(s)=b(t-s, X(s)) d s+\sigma(t-s, X(s)) d W(s), \quad X(0)=x
$$

where $b=\left\{b_{i}\right\}_{i=1}^{d}, \sigma=\left\{\sigma_{i j}\right\}_{i, j=1}^{d}, x \in \mathbb{R}^{d}$ and $t \geq 0$.

This process has two main drawbacks: first, it fails to be an homogeneous strong Markov process and second, the continuity of the flow process does not imply the continuity with respect to $t$. Because of these, we consider 
the augmented process $(\xi(s), X(s))$ defined as the solution to

$$
\begin{aligned}
d \xi(s) & =-d s, \\
d X(s) & =b(\xi(s), X(s)) d s+\sigma(\xi(s), X(s)) d W(s),
\end{aligned}
$$

with $(\xi(0), X(0))=(t, x)$. Throughout this article we will use both processes, $X(s)$ and $(\xi(s), X(s))$, to simplify the exposition.

We assume the following hypotheses on the coefficients $b$ and $\sigma$, denoted by $\mathbf{H 1}$.

\section{H1:}

Let

$$
\begin{aligned}
\sigma(t, x) & :[0, \infty) \times \mathbb{R}^{d} \rightarrow \mathcal{M}\left(\mathbb{R}^{d} \times \mathbb{R}^{d}\right) \\
b(t, x) & :[0, \infty) \times \mathbb{R}^{d} \rightarrow \mathbb{R}^{d}
\end{aligned}
$$

be continuous functions such that

(1) (Continuity.) For all $T>0, n \geq 1$ there exists $L_{1}(T, n)$ such that for all $t, s \in[0, T],\|x\| \leq n,\|y\| \leq n$

- (Locally Lipschitz)

$$
\|\sigma(t, x)-\sigma(t, y)\|+\|b(t, x)-b(t, y)\| \leq L_{1}(T, n)\|x-y\|
$$

- (Locally Hölder)

$$
\|\sigma(t, x)-\sigma(s, x)\|+\|b(t, x)-b(s, x)\| \leq L_{1}(T, n)|t-s|^{\beta},
$$

for some $\beta \in(0,1)$.

(2) (Linear growth.) For each $T>0$, there exists a constant $K(T)$ such that

$$
\|\sigma(t, x)\|^{2}+\|b(t, x)\|^{2} \leq K_{1}(T)^{2}\left(1+\|x\|^{2}\right),
$$

for all $0 \leq t \leq T, x \in \mathbb{R}^{d}$.

(3) (Local ellipticity.) Let $\Sigma \subset \mathbb{R}^{d}$ be a connected, closed set with $C^{3}$ boundary ( $\partial \Sigma$ ). Let $A \subset \mathbb{R}^{d} \backslash \Sigma$ be any bounded open set and $T>0$. There exists $\lambda(T, A)>0$ such that for all $(t, x) \in[0, T] \times \bar{A}$ and $\xi \in \mathbb{R}^{d}$

$$
\sum_{i, j} a_{i j}(t, x) \xi_{i} \xi_{j} \geq \lambda(T, A)\|\xi\|^{2},
$$

where $\left\{a_{i j}\right\}=a=\sigma \sigma^{\prime}$.

Also assume that for all $(t, x) \in[0, T] \times \partial \Sigma$

$$
\begin{gathered}
\sum_{i, j} a_{i j}(t, x) \nu_{i} \nu_{j}=0 \\
\sum_{i} b_{i}(t, x) D_{i} \rho(x)+\sum_{i, j} a_{i, j}(t, x) D_{i j} \rho(x) \geq 0
\end{gathered}
$$

where $\nu$ represents the inward normal and $\rho(x)=\operatorname{dist}(x, \partial \Sigma)$.

Remark 2.1. Let $\hat{b}$ and $\hat{\sigma}$ be the extensions of the functions $b$ and $\sigma$ over the set $\mathbb{R} \times \mathbb{R}^{d}$ defined as

$$
\hat{b}(r, x)= \begin{cases}b(r, x), & \text { if } r \geq 0, \\ b(0, x), & \text { if } r<0,\end{cases}
$$


and

$$
\hat{\sigma}(r, x)= \begin{cases}\sigma(r, x), & \text { if } r \geq 0 \\ \sigma(0, x), & \text { if } r<0\end{cases}
$$

It is easy to prove that the functions $\hat{b}$ and $\hat{\sigma}$ satisfy the locally Lipschitz and Hölder continuity and the linear growth over the set $\mathbb{R} \times \mathbb{R}^{d}$ with the same constants $L_{1}$ and $K_{1}$ defined in $\mathbf{H 1}$.

For the rest of the paper without mentioning it we will allways consider for the functions $b$ and $\sigma$ their respective extensions $\hat{b}$ and $\hat{\sigma}$ and denote them by $b$ and $\sigma$.

Remark 2.2. Condition (5) implies that the process $X(s)$ starting in $x \in \mathbb{R}^{d} \backslash \Sigma$ never reaches the set $\partial \Sigma$ in a finite time, that is, the process $X(s) \in \mathbb{R}^{d} \backslash \Sigma$ for all $s \geq 0$. If the set $\Sigma=\emptyset$ then we are only assuming the local ellipticity in the set $[0, \infty) \times \mathbb{R}^{d}$.

The next proposition presents some of the properties of the process $(\xi(s), X(s))$.

Proposition 2.1. Assume $\mathbf{H 1}$, then the process $(\xi(s), X(s))$ satisfies the following properties:

(1) For all $(t, x) \in[0, \infty) \times \mathbb{R}^{d}$ there exists a unique strong solution $\{(\xi(s), X(s))\}_{s \geq 0}$ to (4).

(2) The flow process $\{(\xi(s ; t), X(s ; x))\}_{s \geq 0,(t, x) \in[0, \infty) \times \mathbb{R}^{d}}$ is continuous a.s..

(3) The process $\{(\xi(s), X(s))\}_{s \geq 0}$ is a strong homogeneous Markov process.

(4) The process $\{(\xi(s), X(s))\}_{s \geq 0}$ does not explode in finite time a.s..

(5) For all $x \in \mathbb{R}^{d}, T>0$ and $r \geq 1$

$$
\mathbb{E}_{x}\left[\sup _{0 \leq s \leq T}\|X(s)\|^{2 r}\right] \leq C\left(T, K_{1}(T), r\right)\left(1+\|x\|^{2 r}\right) .
$$

(6) If $x \in \mathbb{R}^{d} \backslash \Sigma$, then for all $s \geq 0, X(s) \in \mathbb{R} \backslash \Sigma$ a.s.

Proof. See [41] chapter 6 or [32] chapter V for a proof of these properties. Also see Remark 2.1. For a proof of property 6 , see [26] chapter 13 .

\subsubsection{The Cauchy problem.}

Consider the following differential operator

$$
\mathcal{L}[u](t, x)=\sum_{i, j} a_{i j}(t, x) D_{i j} u(t, x)+\sum_{i} b_{i}(t, x) D_{i} u(t, x)
$$

where $D_{i}$ denotes $\frac{\partial}{\partial x_{i}}, D_{i j}$ stands for $\frac{\partial^{2}}{\partial x_{i} \partial x_{j}}$ and $\left\{a_{i j}\right\}=a=\sigma \sigma^{\prime}$. For the rest of this section, we assume $\mathbf{H 1}$ on the coefficients of $\mathcal{L}$. The Cauchy problem for the parabolic equation is

$$
\begin{aligned}
-u_{t}(t, x)+\mathcal{L}[u](t, x)+c(t, x) u(t, x) & =-f(t, x) \quad(t, x) \in(0, \infty) \times \mathbb{R}^{d} \backslash \Sigma, \\
u(0, x) & =h(x) \quad \text { for } x \in \overline{\mathbb{R}^{d} \backslash \Sigma .}
\end{aligned}
$$

We assume the following hypotheses for the data of the Cauchy problem. We denote them by $\mathbf{H 2}$.

H2:

(1) Let

$$
\begin{aligned}
& c(t, x):[0, \infty) \times \mathbb{R}^{d} \rightarrow \mathbb{R} \\
& f(t, x):[0, \infty) \times \mathbb{R}^{d} \rightarrow \mathbb{R},
\end{aligned}
$$

be continuous functions such that 
- (Continuity.) For all $T>0, n \geq 1$ there exists a constant $L_{2}(T, n)$ such that for all $0 \leq s, t \leq T$, $\|x\| \leq n,\|y\| \leq n$

- (Locally Lipschitz)

$$
\|f(t, x)-f(t, y)\|+\|c(t, x)-c(t, y)\| \leq L_{2}(T, n)\|x-y\|,
$$

- (Locally Hölder)

$$
\|f(t, x)-f(s, x)\|+\|c(t, x)-c(s, x)\| \leq L_{2}(T, n)|t-s|^{\beta},
$$

for some $\beta \in(0,1)$.

- (Growth.) There exists $c_{0} \geq 0$ such that

$$
c(t, x) \leq c_{0} \quad \text { for all }(t, x) \in[0, \infty) \times \mathbb{R}^{d} .
$$

For all $T>0$ there exist constants $k>0$ and $K_{2}(T)$ such that

$$
|f(t, x)| \leq K_{2}(T)\left(1+\|x\|^{k}\right),
$$

for all $0 \leq t \leq T, x \in \mathbb{R}^{d}$

(2) Let $h(x): \mathbb{R}^{d} \rightarrow \mathbb{R}$ be a continuous function such that for some $k>0$ and $K_{3}>0$ we have

$$
|h(x)| \leq K_{3}\left(1+\|x\|^{k}\right)
$$

for all $x \in \mathbb{R}^{d}$.

\subsection{Main result.}

The main result for the linear parabolic equation is the following

Theorem 2.1. Assume $\boldsymbol{H} 1$ and $\boldsymbol{H} 2$. Then there exists a unique solution $u \in C\left([0, \infty) \times \overline{\mathbb{R}^{d} \backslash \Sigma}\right) \cap C_{l o c}^{1,2, \beta}\left((0, \infty) \times \mathbb{R}^{d} \backslash \Sigma\right)$ to equation $(7)$. The solution has the representation

$$
u(t, x)=\mathbb{E}_{x}\left[\int_{0}^{t} e^{\int_{0}^{s} c(t-r, X(r)) d r} f(t-s, X(s)) d s+e^{\int_{0}^{t} c(t-r, X(r)) d r} h(X(t))\right],
$$

where $X$ is the solution to the stochastic differential equation

$$
d X(s)=b(t-s, X(s)) d s+\sigma(t-s, X(s)) d W(s), \quad X(0)=x .
$$

Furthermore, for all $T>0$

$$
\sup _{0 \leq t \leq T}|u(t, x)| \leq C\left(T, c_{0}, K_{1}(T), K_{2}(T), K_{3}, k\right)\left(1+\|x\|^{k}\right), \quad x \in \mathbb{R}^{d},
$$

where $c_{0}, K_{1}, K_{2}, K_{3}$ and $k$ are the constants defined in $\boldsymbol{H} \mathbf{1}$ and $\boldsymbol{H} \boldsymbol{2}$.

The proof of this Theorem will be a consequence of several results. To prove it we use probabilistic arguments. Let $v:[0, \infty) \times \mathbb{R}^{d} \rightarrow \mathbb{R}$ be defined as

$$
\begin{aligned}
v(t, x):= & \mathbb{E}_{x}\left[\int_{0}^{t} e^{\int_{0}^{s} c(t-r, X(r)) d r} f(t-s, X(s)) d s\right] \\
& +\mathbb{E}_{x}\left[e^{\int_{0}^{t} c(t-r, X(r)) d r} h(X(t))\right] .
\end{aligned}
$$


Because of $\mathbf{H 2}$ and (6), this function is well defined and finite for $(t, x) \in[0, \infty) \times \mathbb{R}^{d}$. Following some standard arguments (see [18] chapter 4), it can be proved in the case when $v \in C \cap C^{1,2}$ then it solves the Cauchy problem (7). If we assume that all the coefficients, $\sigma, b, c, f$ and $h$, are twice continuously differentiable in $x$, it was proved using the differentiability of the flow process $\{X(s, x)\}$ that the function $v$ is $C^{1,2}$ (see [32] chapter $\mathrm{V}$ for a detailed description of this theory). Additionally, there are explicit formulas for the derivatives of $v$ in terms of the derivatives of the flow. Since we are only assuming the Hölder continuity of the coefficients, the flow is not necessarily differentiable and hence we need a different approach to prove the smoothness of $v$. The next section is devoted to prove the regularity of $v$.

In the rest of this subsection, we assume that $v \in C \cap C^{1,2}$ and prove Theorem 2.1 in that case. The proof is divided in two Lemmas: the first one proves existence, that is, if $v \in C^{1,2}$, then $v$ solves equation (7). The second one is the well known Feynman-Kac's Theorem, that proves that if a classical solution to equation (7) exists, then it has the probabilistic representation given by $v$ and hence is unique.

Lemma 2.1. Assume $\boldsymbol{H} 1$ and $\boldsymbol{H 2}$. Let $v$ be defined as in equation (9). Assume that $v \in C\left([0, \infty) \times \overline{\mathbb{R}^{d} \backslash \Sigma}\right) \times$ $C^{1,2}\left((0, \infty) \times \mathbb{R}^{d} \backslash \Sigma\right)$. Then $v$ fulfils the following equation

$$
\begin{aligned}
-u_{t}(t, x)+\mathcal{L}[u](t, x)+c(t, x) u(t, x) & =-f(t, x) \quad(t, x) \in(0, \infty) \times \mathbb{R}^{d} \backslash \Sigma, \\
u(0, x) & =h(x) \quad \text { for } x \in \overline{\mathbb{R}^{d} \backslash \Sigma} .
\end{aligned}
$$

Furthermore, for all $T>0$

$$
\sup _{0 \leq t \leq T}|v(t, x)| \leq C\left(T, c_{0}, K_{1}(T), K_{2}(T), K_{3}, k\right)\left(1+\|x\|^{k}\right), \quad x \in \mathbb{R},
$$

where $c_{0}, K_{1}, K_{2}$ and $k$ are the constants defined in $\boldsymbol{H} \mathbf{1}$ and $\boldsymbol{H} 2$.

Proof. Let $0<\alpha \leq t$. We have that

$$
\begin{aligned}
& \mathbb{E}_{x}\left[\int_{0}^{t} e^{\int_{0}^{s} c(t-r, X(r)) d r} f(t-s, X(s)) d s+e^{\int_{0}^{t} c(t-r, X(r)) d r} h(X(t)) \mid \mathcal{F}_{\alpha}\right] \\
& =\int_{0}^{\alpha} e^{\int_{0}^{s} c(t-r, X(r)) d r} f(t-s, X(s)) d s+e^{\int_{0}^{\alpha} c(t-r, X(r)) d r} v(t-\alpha, X(\alpha)),
\end{aligned}
$$

(see the argument made for equation (28) in the proof of Theorem 2.3 in section 2.3 below). Since $\int_{0}^{t} e^{\int_{0}^{s} c(t-r, X(r)) d r} f(t-s, X(s)) d s+e^{\int_{0}^{t} c(t-r, X(r)) d r} h(X(t))$ is integrable, then the lefthand side of the last equation is a martingale and so is the righthand side. Since $v \in C^{1,2}\left((0, \infty) \times \mathbb{R}^{d} \backslash \Sigma\right)$ and the process $X(s) \in \mathbb{R}^{d} \backslash \Sigma$ a.s. for all $s \geq 0$ (see Proposition 2.1), then applying Ito's formula to $e^{\int_{0}^{\alpha} c(t-r, X(r)) d r} v(t-\alpha, X(\alpha)$ ) we get

$$
\begin{aligned}
e^{\int_{0}^{\alpha} c(t-r, X(r)) d r} v & (t-\alpha, X(\alpha))=v(x, y) \\
& +\int_{0}^{\alpha} e^{\int_{0}^{s} c(t-r, X(r)) d r}\left(-v_{t}+\mathcal{L}[v]\right)(t-s, X(s)) d s \\
+ & \text { local mgl. }
\end{aligned}
$$

Combining the last two equations we have

$$
\begin{aligned}
e^{\int_{0}^{\alpha} c(t-r, X(r)) d r} & v(t-\alpha, X(\alpha))+\int_{0}^{\alpha} e^{\int_{0}^{s} c(t-r, X(r)) d r} f(t-s, X(s)) d s \\
= & \int_{0}^{\alpha} e^{\int_{0}^{s} c(t-r, X(r)) d r}\left(-v_{t}+\mathcal{L}[v]+f\right)(t-s, X(s)) d s \\
& + \text { local mgl. }
\end{aligned}
$$


Since the lefthand side is a local martingale and the integral on the righthand side is continuous and locally of bounded variation, then for all $s>0$, and $(t, x) \in(0, \infty) \times \mathbb{R}^{d} \backslash \Sigma$

$$
\int_{0}^{\alpha} e^{\int_{0}^{s} c(t-r, X(r)) d r}\left(-v_{t}+\mathcal{L}[v]+f\right)(t-s, X(s)) d s=0 \quad a . s .
$$

This implies that $-v_{t}+\mathcal{L}[v]+f=0$ for all $(t, x) \in(0, \infty) \times \mathbb{R}^{d} \backslash \Sigma$.

The initial condition is fulfilled since $v \in C\left([0, \infty) \times \overline{\mathbb{R}^{d} \backslash \Sigma}\right)$.

Following the same arguments made in the proofs of the Lemmas 2.3 and 2.4 for equations (14) and (20) in section 2.3 below, we can conclude that for all $T>0$

$$
\sup _{0 \leq t \leq T}|v(t, x)| \leq C\left(T, c_{0}, K_{1}(T), K_{2}(T), K_{3}, k\right)\left(1+\|x\|^{k}\right), \quad x \in \mathbb{R}
$$

for some $C$, which concludes the proof.

We enounce a modified version of the Feynman-Kac's Theorem. The proof follows the same lines as the original one, once it is considered that under $\mathbf{H 1}$, the process $X(s) \in \mathbb{R}^{d} \backslash \Sigma$ for all $s \geq 0$. For a proof see [41], Theorem 3.33 in Chapter 6 or [31], Theorem 7.6 in Chapter 5.

Lemma 2.2 (Feynman-Kac's Formula). Assume $\boldsymbol{H 1}$ and H2. Let $W(s)$ be a d-dimensional Brownian motion and let $X(s)$ be the strong solution of the stochastic differential equation

$$
d X(s)=b(t-s, X(s)) d s+\sigma(t-s, X(s)) d W(s), \quad X(0)=x .
$$

Let $u(t, x) \in C^{1,2}\left((0, \infty) \times \mathbb{R}^{d} \backslash \Sigma\right) \cap C\left([0, \infty) \times \overline{\mathbb{R}^{d} \backslash \Sigma}\right)$ be a classical solution to the Cauchy problem

$$
\begin{aligned}
-u_{t}(t, x)+\mathcal{L}[u](t, x)+c(t, x) u(t, x) & =-f(t, x) \quad(t, x) \in(0, \infty) \times \mathbb{R}^{d} \backslash \Sigma, \\
u(0, x) & =h(x) \quad \text { for } x \in \overline{\mathbb{R}^{d} \backslash \Sigma},
\end{aligned}
$$

with $a=\sigma \sigma^{\prime}$ and assume there exists $\mu>0$ such that

$$
\sup _{0 \leq t \leq T}|u(t, x)| \leq K_{T}\left(1+\|x\|^{\mu}\right)
$$

for any $T>0$ and for all $x \in \mathbb{R}$. Then

$$
u(t, x)=\mathbb{E}_{x}\left[\int_{0}^{t} e^{\int_{0}^{s} c(t-r, X(r)) d r} f(t-s, X(s)) d s+e^{\int_{0}^{t} c(t-r, X(r)) d r} h(X(t))\right]
$$

\subsection{Regularity of $v$.}

In this subsection we prove that the function $v$ defined in equation (9) belongs to the space $C([0, \infty) \times$ $\left.\overline{\mathbb{R}^{d} \backslash \Sigma}\right) \cap C_{\text {loc }}^{1,2, \beta}\left((0, \infty) \times \mathbb{R}^{d} \backslash \Sigma\right)$. To this end, we use the theory of stochastic differential equations and the theory of parabolic equations with Hölder coefficients in bounded domains. We first prove that $v$ is continuous in $[0, \infty) \times \overline{\mathbb{R}^{d} \backslash \Sigma}$ using the properties of the paths of the stochastic process $X(t)$. Then, to prove $C^{1,2}$ regularity, we observe that on any smooth, bounded subset $\left[T_{0}, T_{1}\right] \times A \subset[0, \infty) \times \mathbb{R}^{d} \backslash \Sigma, v$ fulfils a parabolic differential equation for which a classical solution exists, thanks to the theory of parabolic equations. This entails that $v \in C_{\mathrm{loc}}^{1,2, \beta}\left((0, \infty) \times \mathbb{R}^{d} \backslash \Sigma\right)$. 
2.3.1. Continuity of $v$.

We can write $v$ as

$$
\begin{aligned}
v(t, x)= & \mathbb{E}_{t, x}\left[\int_{0}^{t} e^{\int_{0}^{s} c(\xi(r), X(r)) d r} f(\xi(s), X(s)) d s\right] \\
& +\mathbb{E}_{t, x}\left[e^{\int_{0}^{t} c(\xi(r), X(r)) d r} h(X(t))\right]
\end{aligned}
$$

where the process $\{\xi(s), X(s)\}$ is the solution to equation (4). To prove that $v$ is continuous in $[0, \infty) \times \mathbb{R}^{d}$ we rewrite it as $v=v_{1}+v_{2}$ where

$$
v_{1}(t, x):=\mathbb{E}_{t, x}\left[\int_{0}^{t} e^{\int_{0}^{s} c(\xi(r), X(r)) d r} f(\xi(s), X(s)) d s\right]
$$

and

$$
v_{2}(t, x):=\mathbb{E}_{t, x}\left[e^{\int_{0}^{t} c(\xi(r), X(r)) d r} h(X(t))\right]
$$

We have the following Theorem.

Theorem 2.2. Assume $\boldsymbol{H} 1$ and $\boldsymbol{H} 2$. Then the function $v$ defined in equation (11) is continuous in $[0, \infty) \times$ $\overline{\mathbb{R}^{d} \backslash \Sigma}$.

The proof is divided in two Lemmas.

Lemma 2.3. Assume $\boldsymbol{H} 1$ and $\boldsymbol{H} 2$. Then $v_{1}$ defined as in equation (12) is a continuous function over $[0, \infty) \times$ $\overline{\mathbb{R}^{d} \backslash \Sigma}$.

Proof. We first prove the continuity in $(0, \infty) \times \overline{\mathbb{R}^{d} \backslash \Sigma}$. Let $\left\{\left\{\left(t_{n}, x_{n}\right)\right\}_{n \in \mathbb{N}},(t, x)\right\} \subset(0, \infty) \times \overline{\mathbb{R}^{d} \backslash \Sigma}$. Assume that $\left(t_{n}, x_{n}\right) \underset{n \rightarrow \infty}{\longrightarrow}(t, x)$. We need to prove that

$$
v_{1}\left(t_{n}, x_{n}\right) \underset{n \rightarrow \infty}{\longrightarrow} v_{1}(t, x)
$$

We will prove that for any $\epsilon>0$ there exists a $N \in \mathbb{N}$ such that for any $n \geq N$

$$
\left|v_{1}\left(t_{n}, x_{n}\right)-v_{1}(t, x)\right|<\epsilon
$$

Let $\epsilon>0,0<\alpha \ll 1$ and $N_{1} \in \mathbb{N}$ such that

$$
\left\|\left(t_{n}, x_{n}\right)-(t, x)\right\|<\alpha \quad \text { for } n \geq N_{1} .
$$

Then if $n \geq N_{1}$ we get $t_{n} \leq t+\alpha$ and $\left\|x_{n}\right\| \leq\|x\|+\alpha$.

Let $\left(\xi_{n}, X_{n}\right)$ and $(\xi, X)$ denote the solutions to equation (4) with initial conditions $\left(t_{n}, x_{n}\right)$ and $(t, x)$ respectively. We prove first that for all $n \geq N_{1}$, the random variables

$$
\begin{aligned}
Y_{n}:= & \mid \int_{0}^{t_{n}} e^{\int_{0}^{s} c\left(\xi_{n}(r), X_{n}(r)\right)} f\left(\xi_{n}(s), X_{n}(s)\right) d s \\
& -\int_{0}^{t} e^{\int_{0}^{s} c(\xi(r), X(r)) d r} f(\xi(s), X(s)) d s \mid
\end{aligned}
$$


are uniformly integrable. To prove this we observe that

$$
\begin{aligned}
\int_{\Omega} Y_{n}^{2} d \mathbb{P} \leq & 2 \int_{\Omega}\left(\int_{0}^{t_{n}} e^{\int_{0}^{s} c\left(\xi_{n}(r), X_{n}(r)\right) d r} f\left(\xi_{n}(s), X_{n}(s)\right) d s\right)^{2} d \mathbb{P} \\
& +2 \int_{\Omega}\left(\int_{0}^{t} e^{\int_{0}^{s} c(\xi(r), X(r)) d r} f(\xi(s), X(s)) d s\right)^{2} d \mathbb{P} \\
& \leq 2 \int_{\Omega}\left(\int_{0}^{t_{n}} e^{c_{0} s} K_{2}(t+\alpha)\left(1+\left\|X_{n}(s)\right\|^{k}\right) d s\right)^{2} d \mathbb{P}+C_{t, x} \\
\leq & 4(t+\alpha)^{2} e^{2 c_{0}(t+\alpha)} K_{2}^{2}(t+\alpha)\left(1+\int_{\Omega} \sup _{0 \leq s \leq t+\alpha}\left\|X_{n}(s)\right\|^{2 k} d \mathbb{P}\right)+C_{t, x} \\
& \leq C(t, \alpha, k)\left(1+\left\|x_{n}\right\|^{4 k}\right)+C_{t, x} \\
& \leq C(t, \alpha, k)\left(1+(\|x\|+\alpha)^{4 k}\right)+C_{t, x}<\infty
\end{aligned}
$$

due to $\mathbf{H 2}$ and equation (6). It follows from Theorem 4.2 page 215 in [27], that $\left\{Y_{n}\right\}_{n \geq N_{1}}$ is uniformly integrable. Let $0<\eta<1$ and $M>0$. Define $M_{1}:=1+M$ and $A_{1}:=[0, t+\alpha] \times\left[-M_{1}, M_{1}\right]^{d}$. Then

$$
\begin{aligned}
\left|v_{1}\left(t_{n}, x_{n}\right)-v_{1}(t, x)\right| \leq & \int_{\left\{\|X\|_{t+\alpha} \leq M\right\} \cap\left\{\left\|X_{n}-X\right\|_{t+\alpha} \leq \eta\right\}}\left|Y_{n}\right| d \mathbb{P} \\
& +\int_{\Omega \backslash\left(\left\{\|X\|_{t+\alpha} \leq M\right\} \cap\left\{\left\|X_{n}-X\right\|_{t+\alpha} \leq \eta\right\}\right)}\left|Y_{n}\right| d \mathbb{P} .
\end{aligned}
$$

As a consequence of the uniformly integrability, for every $\epsilon>0$ there exists a $\delta(\epsilon)>0$ such that $\sup _{n} \int_{B}\left|Y_{n}\right| d \mathbb{P}<$ $\frac{\epsilon}{2}$ for all $B \in \mathcal{F}$ such that $\mathbb{P}[B] \leq \delta(\epsilon)$.

By Proposition 2.1 we may choose $M>0$ such that

$$
\mathbb{P}\left[\|X\|_{t+\alpha}>M\right] \leq \frac{\delta(\epsilon)}{2}
$$

and $N_{2} \in \mathbb{N}$ for which

$$
\mathbb{P}\left[\left\|X_{n}-X\right\|_{t+\alpha}>\eta\right] \leq \frac{\delta(\epsilon)}{2}
$$

for all $n \geq N_{2}$ (using Theorem 5.1 in Section 5). So for all $n \geq N_{1} \vee N_{2}$ we get

$$
\left|v_{1}\left(t_{n}, x_{n}\right)-v_{1}(t, x)\right| \leq \int_{\left\{\|X\|_{t+\alpha} \leq M\right\} \cap\left\{\left\|X_{n}-X\right\|_{t+\alpha} \leq \eta\right\}}\left|Y_{n}\right| d \mathbb{P}+\frac{\epsilon}{2}
$$

Now, on the set $B_{M, n, \eta}:=\left\{\|X\|_{t+\alpha} \leq M\right\} \cap\left\{\left\|X_{n}-X\right\|_{t+\alpha} \leq \eta\right\}$ we have that $\left(\xi_{n}(s), X_{n}(s)\right),\left(\xi(s), X_{n}(s)\right) \in A_{1}$. So

$$
\begin{aligned}
\int_{B_{M, n, \eta}}\left|Y_{n}\right| d \mathbb{P} \leq & \int_{B_{M, n, \eta}} \int_{0}^{t_{n} \wedge t} \mid e^{\int_{0}^{s} c\left(\xi_{n}(r), X_{n}(r)\right) d r} f\left(\xi_{n}(s), X_{n}(s)\right) \\
& -e^{\int_{0}^{s} c(\xi(r), X(r)) d r} f(\xi(s), X(s)) \mid d s d \mathbb{P} \\
+ & \int_{B_{M, n, \eta}} \int_{t_{n} \wedge t}^{t_{n} \vee t} \mid e^{\int_{0}^{s} c\left(\xi_{n}(r), X_{n}(r)\right) d r} f\left(\xi_{n}(s), X_{n}(s)\right) \mathbb{1}_{t_{n}>t} \\
& +e^{\int_{0}^{s} c(\xi(r), X(r)) d r} f(\xi(s), X(s)) \mathbb{1}_{t_{n} \leq t} \mid d s d \mathbb{P} .
\end{aligned}
$$


For (17) we have

$$
\begin{aligned}
(17) & \leq \int_{B_{M, n, \eta}} \int_{t_{n} \wedge t}^{t_{n} \vee t} e^{c_{0} s} K_{f}\left(A_{1}\right) d s d \mathbb{P} \\
& \leq e^{c_{0}(t+\alpha)} K_{f}\left(A_{1}\right)\left|t_{n}-t\right| \\
& \leq e^{c_{0}(t+\alpha)} K_{f}\left(A_{1}\right)\left|t_{n}-t\right|^{\beta}
\end{aligned}
$$

since $\left|t_{n}-t\right|<\alpha \ll 1$. Next we study (16). We get that

$$
\begin{aligned}
&(16) \leq \int_{B_{M, n, \eta}} \int_{0}^{t_{n} \wedge t} e^{\int_{0}^{s} c\left(\xi_{n}(r), X_{n}(r)\right) d r} \\
& \times\left|f\left(\xi_{n}(s), X_{n}(s)\right)-f(\xi(s), X(s))\right| d s d \mathbb{P} \\
&+\quad \int_{B_{M, n, \eta}} \int_{0}^{t_{n} \wedge t}|f(\xi(s), X(s))| \\
& \quad \times\left|e^{\int_{0}^{s} c\left(\xi_{n}(r), X_{n}(r)\right) d r}-e^{\int_{0}^{s} c(\xi(r), X(r)) d r}\right| d s d \mathbb{P} .
\end{aligned}
$$

We analyze the two terms in the righthand side separately. For the first part, since $f$ is locally Lipschitz, we get

$$
\begin{aligned}
(18) & \leq \int_{B_{M, n, \eta}} \int_{0}^{t_{n} \wedge t} e^{c_{0} s} L_{f}\left(A_{1}\right)\left(\left|t_{n}-t\right|^{\beta}+\left\|X_{n}(s)-X(s)\right\|\right) d s d \mathbb{P} \\
& \leq e^{c_{0}(t+\alpha)}(t+\alpha) L_{f}\left(A_{1}\right)\left(\left|t_{n}-t\right|^{\beta}+\eta\right) .
\end{aligned}
$$

It follows from the locally Lipschitz continuity of $c$, that on $B_{M, n, \eta}$

$$
\begin{aligned}
\mid e^{\int_{0}^{s} c\left(\xi_{n}(r), X_{n}(r)\right) d r}-e^{\int_{0}^{s} c(\xi(r), X(r)) d r \mid}=e^{\int_{0}^{s} c(\xi(r), X(r)) d r} & \times\left|\exp \left\{\int_{0}^{s}\left(c\left(\xi_{n}(r), X_{n}(r)\right)-c(\xi(r), X(r))\right) d r\right\}-1\right| \\
\leq & e^{c_{0} s}\left(\exp \left\{\int_{0}^{s}\left|c\left(\xi_{n}(r), X_{n}(r)\right)-c(\xi(r), X(r))\right| d r\right\}-1\right) \\
\leq & e^{c_{0} s}\left(\exp \left\{\int_{0}^{s} L_{c}\left(A_{1}\right)\left(\left|t_{n}-t\right|^{\beta}+\left\|X_{n}(r)-X(r)\right\|\right) d r\right\}-1\right) \\
\leq & e^{c_{0} s}\left(\exp \left\{L_{c}\left(A_{1}\right) s\left(\left|t_{n}-t\right|^{\beta}+\eta\right)\right\}-1\right),
\end{aligned}
$$

since $\left|e^{x}-1\right| \leq e^{|x|}-1$. If we choose $N_{3} \in \mathbb{N}$ such that $\left|t_{n}-t\right|^{\beta} \leq \frac{1}{2 L_{c}\left(A_{1}\right)(t+\alpha)}$ for all $n \geq N_{3}$ and $\eta \leq \frac{1}{2 L_{c}\left(A_{1}\right)(t+\alpha)}$, we get by the Mean Value Theorem that

$$
\left|e^{\int_{0}^{s} c\left(\xi_{n}(r), X_{n}(r)\right) d r}-e^{\int_{0}^{s} c(\xi(r), X(r)) d r}\right| \leq e^{c_{0} s} e L_{c}\left(A_{1}\right) s\left(\left|t_{n}-t\right|^{\beta}+\eta\right) .
$$

So for (19) we get the following bound

$$
\begin{aligned}
(19) & \leq \int_{B_{M, n, \eta}} \int_{0}^{t_{n} \wedge t} K_{f}\left(A_{1}\right) e^{c_{0} s} e L_{c}\left(A_{1}\right) s\left(\left|t_{n}-t\right|^{\beta}+\eta\right) d s d \mathbb{P} \\
& \leq K_{f}\left(A_{1}\right) e^{c_{0}(t+\alpha)} e L_{c}\left(A_{1}\right)(t+\alpha)^{2}\left(\left|t_{n}-t\right|^{\beta}+\eta\right) .
\end{aligned}
$$


Hence to prove continuity we chose the parameters in the following order: let $\epsilon>0,0<\alpha \ll 1, \delta(\epsilon), M>0$. Then let $\eta$

$$
\begin{aligned}
\eta<\min & \left\{1, \frac{1}{2 L_{c}\left(A_{1}\right)(t+\alpha)}, \frac{\epsilon}{16 e^{c_{0}(t+\alpha)}(t+\alpha) L_{f}\left(A_{1}\right)}\right. \\
& \left.\frac{\epsilon}{16 K_{f}\left(A_{1}\right) e^{c_{0}(t+\alpha)} e L_{c}\left(A_{1}\right)(t+\alpha)}\right\} .
\end{aligned}
$$

And let $N_{3} \in \mathbb{N}$ be such that

$$
\begin{aligned}
\left|t_{n}-t\right|^{\beta}<\min & \left\{\frac{1}{2 L_{c}\left(A_{1}\right)(t+\alpha)}, \frac{\epsilon}{4 e^{c_{0}(t+\alpha)} K_{f}\left(A_{1}\right)}\right. \\
& , \frac{\epsilon}{16 e^{c_{0}(t+\alpha)}(t+\alpha) L_{f}\left(A_{1}\right)} \\
& \left., \frac{\epsilon}{16 K_{f}\left(A_{1}\right) e^{c_{0}(t+\alpha)} e L_{c}\left(A_{1}\right)(t+\alpha)}\right\} .
\end{aligned}
$$

for all $n \geq N_{3}$

So if $N=N_{1} \vee N_{2} \vee N_{3}$ we have that for all $n \geq N$

$$
\left|v_{1}\left(t_{n}, x_{n}\right)-v_{1}(t, x)\right|<\epsilon
$$

Therefore $v_{1}$ is continuous in $(0, \infty) \times \overline{\mathbb{R}^{d} \backslash \Sigma}$.

For the continuity at $t=0$ we proceed the same way. Let $\left(t_{n}, x_{n}\right) \rightarrow(0, x), \alpha>0$ and $N_{1} \geq 1$ be such that

$$
\left\|\left(t_{n}, x_{n}\right)-(0, x)\right\| \leq \alpha
$$

for all $n \geq N_{1}$. Since $v_{1}(0, x) \equiv 0$, we need to prove that

$$
\left|v_{1}\left(t_{n}, x_{n}\right)\right| \underset{n \rightarrow \infty}{\longrightarrow} 0
$$

which is a consequence of the following

$$
\begin{aligned}
\left|v_{1}\left(t_{n}, x_{n}\right)\right| & \leq \mathbb{E}_{t_{n}, x_{n}}\left[\int_{0}^{t_{n}} e^{\int_{0}^{s} c\left(\xi_{n}(r), X_{n}(r)\right) d r}\left|f\left(\xi_{n}(s), X_{n}(s)\right)\right| d s\right] \\
& \leq e^{c_{0} \alpha} \int_{0}^{t_{n}} \mathbb{E}_{t_{n}, x_{n}}\left[\left|f\left(\xi_{n}(s), X_{n}(s)\right)\right|\right] d s \\
& \leq e^{c_{0} \alpha} \int_{0}^{t_{n}} \mathbb{E}_{x_{n}}\left[K(\alpha)\left(1+\left\|X_{n}(s)\right\|^{k}\right)\right] d s \\
& \leq e^{c_{0} \alpha} \int_{0}^{t_{n}} K_{2}(\alpha)\left(1+\mathbb{E}_{x_{n}}\left[\sup _{0 \leq r \leq \alpha}\left\|X_{n}(r)\right\|^{k}\right]\right) d s \\
& \leq e^{c_{0} \alpha} \int_{0}^{t_{n}} K_{2}(\alpha)\left(1+C(\alpha, k)\left(1+\left\|x_{n}\right\|^{k}\right)\right) d s \\
& \leq e^{c_{0} \alpha} K_{2}(\alpha)\left(1+C(\alpha, k)\left(1+(\|x\|+\alpha)^{k}\right)\right) t_{n} \underset{n \rightarrow \infty}{\longrightarrow} 0 .
\end{aligned}
$$

Lemma 2.4. Assume $\boldsymbol{H} 1$ and $\boldsymbol{H} 2$. Then $v_{2}$ defined as in equation (13) is a continuous function over $[0, \infty) \times$ $\overline{\mathbb{R}^{d} \backslash \Sigma}$. 
Proof. We proceed in the same way as in the proof of Lemma 2.3. Since the proof follows essentially the same lines we only present an sketch of it. Let $\left\{\left(t_{n}, x_{n}\right)\right\}_{n \in \mathbb{N}},(t, x), \alpha,\left(\xi_{n}, X_{n}\right)$ and $(\xi, X)$ be as in Lemma 2.3. First we prove that the sequence of random variables

$$
Y_{n}:=\left|e^{\int_{0}^{t_{n}} c\left(\xi_{n}(r), X_{n}(r)\right) d r} h\left(X_{n}\left(t_{n}\right)\right)-e^{\int_{0}^{t} c(\xi(r), X(r)) d r} h(X(t))\right|
$$

is uniformly integrable for all $n \geq N_{1}$. As in equation (14) we can prove that

$$
\int_{\Omega} Y_{n}^{2} d \mathbb{P} \leq C(t, \alpha, r)\left(1+(\|x\|+\alpha)^{4 r}\right)+C_{t, x}<\infty
$$

Let $M>0,0<\eta<1$ and $\epsilon>0$. Let $M_{1}, A_{1}$ and $\delta(\epsilon)$ be as in Lemma 2.3. Hence we can prove that

$$
\left|v_{2}\left(t_{n}, x_{n}\right)-v_{2}(t, x)\right| \leq \int_{\left\{\|X\|_{t+\alpha} \leq M\right\} \cap\left\{\left\|X_{n}-X\right\|_{t+\alpha} \leq \eta\right\}}\left|Y_{n}\right| d \mathbb{P}+\frac{\epsilon}{2} .
$$

Let $B_{M, n, \eta}:=\left\{\|X\|_{t+\alpha} \leq M\right\} \cap\left\{\left\|X_{n}-X\right\|_{t+\alpha} \leq \eta\right\}$. Then

$$
\begin{aligned}
\int_{B_{M, n, \eta}} Y_{n} d \mathbb{P} & \leq \int_{B_{M, n, \eta}} e^{\int_{0}^{t_{n}} c\left(\xi_{n}(r), X_{n}(r)\right) d r}\left|h\left(X_{n}\left(t_{n}\right)\right)-h(X(t))\right| d \mathbb{P} \\
& +\int_{B_{M, n, \eta}}|h(X(t))| \\
& \times\left|e^{\int_{0}^{t_{n}} c\left(\xi_{n}(r), X_{n}(r)\right) d r}-e^{\int_{0}^{t} c(\xi(r), X(r)) d r}\right| d \mathbb{P}
\end{aligned}
$$

We begin with the second part of the righthand side of the last equation. As in equation (19) we can prove that

$$
\begin{aligned}
(22) \leq & K_{h}\left(A_{1}\right) e^{c_{0}(t+\alpha)} e \\
& \times\left(\left(L_{c}\left(A_{1}\right)(t+\alpha)+K_{c}\left(A_{1}\right)\right)\left|t_{n}-t\right|^{\beta}+L_{c}\left(A_{1}\right)(t+\alpha) \eta\right) .
\end{aligned}
$$

For (21) we have that

$$
\begin{aligned}
(21) \leq & e^{c_{0}(t+\alpha)} \int_{B_{M, n, \eta}}\left|h\left(X_{n}\left(t_{n}\right)\right)-h\left(X\left(t_{n}\right)\right)\right| d \mathbb{P} \\
& \left.+e^{c_{0}(t+\alpha)} \int_{B_{M, n, \eta}}\left|h\left(X\left(t_{n}\right)\right)-h(X(t))\right|\right) d \mathbb{P}
\end{aligned}
$$

We first analyze (23). Since $h$ is continuous, then it is uniformly continuous over the compact set $A_{1}$. Then, for $\epsilon>0$, there exists $\gamma\left(\epsilon, M, c_{0}, \alpha, t\right)$ such that if $\|x-y\|<\gamma\left(\epsilon, M, c_{0}, \alpha, t\right)$, then $|h(x)-h(y)|<\frac{\epsilon}{8 e^{c_{0}(t+\alpha)}}$ for all $x, y \in A_{1}$. So for $\eta<\gamma\left(\epsilon, M, c_{0}, \alpha, t\right)$, over $B_{M, n, \eta}$

$$
\left|h\left(X_{n}\left(t_{n}\right)\right)-h\left(X\left(t_{n}\right)\right)\right|<\frac{\epsilon}{8 e^{c_{0}(t+\alpha)}} .
$$

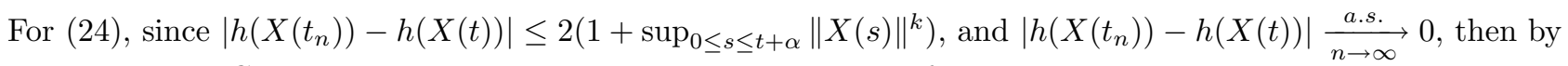
the Dominated Convergence Theorem, there exists $N_{3}$ such that for all $n \geq N_{3}$

$$
\int_{\Omega}\left|h\left(X\left(t_{n}\right)\right)-h(X(t))\right| d \mathbb{P}<\frac{\epsilon}{8 e^{c_{0}(t+\alpha)}} .
$$


So to prove continuity we proceed as in the proof of Lemma 2.3 with

$$
\begin{aligned}
\eta<\min & \left\{1, \gamma\left(\epsilon, M, c_{0}, \alpha, t\right), \frac{1}{2 L_{c}\left(A_{1}\right)(t+\alpha)}\right. \\
& \left., \frac{\epsilon}{8 K_{h}\left(A_{1}\right) e^{c_{0}(t+\alpha)} e L_{c}\left(A_{1}\right)(t+\alpha)}\right\} .
\end{aligned}
$$

and $N_{3} \in \mathbb{N}$ be such that

$$
\begin{aligned}
\left|t_{n}-t\right|^{\beta}<\min & \left\{\frac{1}{2\left(L_{c}\left(A_{1}\right)(t+\alpha)+K_{c}\left(A_{1}\right)\right)}\right. \\
& \left., \frac{\epsilon}{8 K_{h}\left(A_{1}\right) e^{c_{0}(t+\alpha)} e\left(\left(L_{c}\left(A_{1}\right)(t+\alpha)+K_{c}\left(A_{1}\right)\right)\right.}\right\} .
\end{aligned}
$$

for all $n \geq N_{3}$. And we conclude that $v_{2}$ is continuous over $(0, \infty) \times \overline{\mathbb{R}^{d} \backslash \Sigma}$.

The continuity at $t=0$ is treated in a similar way and hence the proof is complete.

\subsubsection{Differentiability of $v$.}

To prove that $v$ is a solution of equation (7), we need to prove that $v \in C^{1,2}\left((0, \infty) \times \mathbb{R}^{d} \backslash \Sigma\right)$. Let $0 \leq T_{0}<T_{1}$ and $A \subset \mathbb{R}^{d} \backslash \Sigma$ be a bounded open set with $C^{2}$ boundary. Consider the following parabolic differential equation

$$
\begin{gathered}
-u_{t}(t, x)+\mathcal{L}[u](t, x)+c(t, x) u(t, x)=-f(t, x) \quad(t, x) \in\left[T_{0}, T_{1}\right] \times A, \\
u\left(T_{0}, x\right)=v\left(T_{0}, x\right) \quad \text { for } x \in A, \\
u(t, x)=v(t, x) \quad \text { for }(t, x) \in\left(T_{0}, T_{1}\right] \times \partial A .
\end{gathered}
$$

where the boundary data is $v$. From H1, H2 and the continuity of $v$, the existence of a classical solution to equation (26) follows from Theorem 5.2 in Section 5.

We can prove the following Theorem

Theorem 2.3. Assume $\boldsymbol{H} 1$ and $\boldsymbol{H} 2$. Let $v$ be defined as in equation $(9)$. Then $v \in C_{l o c}^{1,2, \beta}\left((0, \infty) \times \mathbb{R}^{d} \backslash \Sigma\right)$.

Proof. Let $w$ be the solution to equation (26). Define the following stopping times

$$
\begin{aligned}
\tau_{T} & :=\inf \left\{s>0 \mid \xi(s)<T_{0}\right\} \\
\tau_{A} & :=\inf \{s>0 \mid X(s) \notin \bar{A}\}, \\
\tau & :=\tau_{T} \wedge \tau_{A} .
\end{aligned}
$$

Following the same arguments of Section 5 in Chapter 6 of [25], we can prove that $w$ has the following representation

$$
\begin{aligned}
w(t, x)= & \mathbb{E}_{x}\left[\int_{0}^{\tau} e^{\int_{0}^{s} c(t-r, X(r)) d r} f(t-s, X(s)) d s\right] \\
& +\mathbb{E}_{x}\left[e^{\int_{0}^{\tau} c(t-r, X(r)) d s} v(t-\tau, X(\tau))\right]
\end{aligned}
$$

Using the strong Markov property of the process $(\xi(s), X(s))$ we prove the following equality for $v$

$$
\begin{aligned}
v(t, x)= & \mathbb{E}_{t, x}\left[\int_{0}^{\tau} e^{\int_{0}^{s} c(\xi(r), X(r)) d r} f(\xi(s), X(s)) d s\right] \\
& +\mathbb{E}_{t, x}\left[e^{\int_{0}^{\tau} c(\xi(r), X(r)) d s} v(\xi(\tau), X(\tau))\right] .
\end{aligned}
$$


Let $v_{1}$ and $v_{2}$ be defined as in equations (12) and (13). For $v_{1}$

$$
\begin{aligned}
v_{1}(t, x)= & \mathbb{E}_{t, x}\left[\mathbb{E}\left[\int_{0}^{t} e^{\int_{0}^{s} c(\xi(r), X(r)) d r} f(\xi(s), X(s)) d s \mid \mathcal{F}_{\tau}\right]\right] \\
= & \mathbb{E}_{t, x}\left[\mathbb{E}\left[\int_{0}^{\tau} e^{\int_{0}^{s} c(\xi(r), X(r)) d r} f(\xi(s), X(s)) d s \mid \mathcal{F}_{\tau}\right]\right] \\
& +\mathbb{E}_{t, x}\left[\mathbb{E}\left[\int_{\tau}^{t} e^{\int_{0}^{s} c(\xi(r), X(r)) d r} f(\xi(s), X(s)) d s \mid \mathcal{F}_{\tau}\right]\right]
\end{aligned}
$$

Now,

$$
(29)=\mathbb{E}_{t, x}\left[\int_{0}^{\tau} e^{\int_{0}^{s} c(\xi(r), X(r)) d r} f(\xi(s), X(s)) d s\right] .
$$

We make a couple of changes of variable in (30) to get

$$
\begin{aligned}
(30) & =\mathbb{E}_{t, x}\left[\mathbb{E}\left[\int_{0}^{t-\tau} e^{\int_{0}^{s+\tau} c(\xi(r), X(r)) d r} f(\xi(s+\tau), X(s+\tau)) d s \mid \mathcal{F}_{\tau}\right]\right] \\
& =\mathbb{E}_{t, x}\left[e^{\int_{0}^{\tau} c(\xi(r), X(r)) d r} \mathbb{E}\left[\int_{0}^{t-\tau} e^{\int_{0}^{s} c(\xi(r+\tau), X(r+\tau)) d r} f(\xi(s+\tau), X(s+\tau)) d s \mid \mathcal{F}_{\tau}\right]\right] .
\end{aligned}
$$

Since $(\xi(s), X(s))$ is a strong Markov process we get that

$$
\begin{aligned}
(30) & =\mathbb{E}_{t, x}\left[e^{\int_{0}^{\tau} c(\xi(r), X(r)) d r} \mathbb{E}_{\xi(\tau), X(\tau)}\left[\int_{0}^{t-\tau} e^{\int_{0}^{s} c(\xi(r), X(r)) d r} f(\xi(s), X(s)) d s\right]\right] \\
& =\mathbb{E}_{t, x}\left[e^{\int_{0}^{\tau} c(\xi(r), X(r)) d r} v_{1}(\xi(\tau), X(\tau))\right] .
\end{aligned}
$$

So we conclude that

$$
\begin{aligned}
v_{1}(t, x)= & \mathbb{E}_{t, x}\left[\int_{0}^{\tau} e^{\int_{0}^{s} c(\xi(r), X(r)) d r} f(\xi(s), X(s)) d s\right] \\
& +\mathbb{E}_{t, x}\left[e^{\int_{0}^{\tau} c(\xi(r), X(r)) d r} v_{1}(\xi(\tau), X(\tau))\right] .
\end{aligned}
$$

For $v_{2}$ we make a couple of changes of variable to get

$$
\begin{aligned}
v_{2}(t, x) & =\mathbb{E}_{t, x}\left[\mathbb{E}\left[e^{\int_{0}^{t} c(\xi(r), X(r)) d r} h(X(t)) \mid \mathcal{F}_{\tau}\right]\right] \\
& =\mathbb{E}_{t, x}\left[e^{\int_{0}^{\tau} c(\xi(r), X(r)) d r} \mathbb{E}\left[e^{\int_{0}^{t-\tau} c(\xi(r+\tau), X(r+\tau)) d r} h(X(t-\tau+\tau)) \mid \mathcal{F}_{\tau}\right]\right] \\
& =\mathbb{E}_{t, x}\left[e^{\int_{0}^{\tau} c(\xi(r), X(r)) d r} \mathbb{E}_{\xi(\tau), X(\tau)}\left[e^{\int_{0}^{t-\tau} c(\xi(r), X(r)) d r} h(X(t-\tau))\right]\right] \\
& =\mathbb{E}_{t, x}\left[e^{\int_{0}^{\tau} c(\xi(r), X(r)) d r} v_{2}(\xi(\tau), X(\tau))\right] .
\end{aligned}
$$

Thanks to equations (31) and (32) we conclude that equation (28) is true.

So due to equations (27) and (28) we have that $v=w$. Since $w \in C^{1,2, \beta}\left(\left(T_{0}, T_{1}\right) \times A\right)$ (see Theorem 5.2 below) and $T_{0} T_{1}$ and $A$ are arbitrary we get that $v \in C_{\mathrm{loc}}^{1,2, \beta}\left((0, \infty) \times \mathbb{R}^{d} \backslash \Sigma\right)$ and the proof is complete.

We are ready to prove the Main Theorem.

Proof of Theorem 2.1. Let $v$ be defined as in equation (9). Thanks to Theorems 2.2 and 2.3 and Lemmas 2.1 and 2.2 the proof is complete. 
Remark 2.3. The methods used in this paper can be made under broader assumptions on the coefficients of $\mathcal{L}$, considering a condition of Lyapunov type. Let $\varphi \in C^{1,2}\left((0, T) \times \mathbb{R}^{d}\right)$ be such that

$$
\lim _{|x| \rightarrow \infty} \inf _{0 \leq t \leq T} \varphi(t, x)=\infty
$$

and for some $\lambda>0$

$$
\sup _{[0, T] \times \mathbb{R}^{d}}\left\{\left(-\frac{\partial}{\partial t}+\mathcal{L}\right) \varphi(t, x)-\lambda \varphi(t, x)\right\}<\infty .
$$

Thanks to Theorem 10.2.1 in [40], we have that under these assumptions the martingale problem is well-posed and so there exists a unique weak solution to equation (3). It follows from the locally Lipschitz continuity in $x$, of $\sigma$ and $b$, that the existence is pathwise unique (see Corollary 2.9 on Chapter 6 in [41]). This and the Yamada-Watanabe's Theorem (Theorem 1.25, Chapter 6 in [41]) imply the existence of a unique strong solution to equation (3). Finally, due to Theorem 38 of Chapter $\mathrm{V}$ in [38] we have that the flow process $X(t ; x)$ is continuous. Despite this, it is not clear how to control the growth of the moments (see equation (6)) and hence we need to work with bounded data $\left(f, g \in C_{b}\right)$ in order to repeat the arguments made in the proof of Theorem 2.2. Many interesting stochastic control problems need the non-boundedness of the data and since our motivations came from that field, we assume $\mathbf{H 1}$ in order to have estimations like (6) for the moments of the process. In section 4 , we present an example that illustrates this.

\section{Semilinear parabolic equations.}

We consider the following differential problem

$$
\begin{array}{r}
-u_{t}(t, x)+\sum_{i j} a_{i j}(t, x) D_{i j} u(t, x) \\
+\sup _{\alpha \in \Lambda}\left\{\mathcal{L}_{1}^{\alpha}[u](t, x)+f(t, x, \alpha)\right\}=0, \quad \text { in }(0, \infty) \times \mathbb{R}^{d} \\
u(0, x)=h(x), \quad x \in \mathbb{R}^{d},
\end{array}
$$

where $a=\sigma \sigma^{\prime}$ and

$$
\mathcal{L}_{1}^{\alpha}[u](t, x):=\sum_{i} b_{i}(t, x, \alpha) D_{i} u(t, x)+c(t, x, \alpha) u(t, x) .
$$

We assume the following hypotheses on the coefficients, we denote them by $\mathbf{H 3}$.

H3:

(1) $\Lambda \subset \mathbb{R}^{m}$ is a compact set.

(2) $\sigma$ follows the same hypotheses made in $\mathbf{H 1}$ with $\Sigma=\emptyset$.

(3) Let $h(x): \mathbb{R}^{d} \rightarrow \mathbb{R}$ be a locally Hölder continuous function of order $\beta$ such that for some $k>0$ and $K_{3}>0$ we have

for all $x \in \mathbb{R}^{d}$.

$$
|h(x)| \leq K_{3}\left(1+\|x\|^{k}\right)
$$

(4) Let

$$
\begin{aligned}
& b:[0, \infty) \times \mathbb{R}^{d} \times \Lambda \rightarrow \mathbb{R}^{d} \\
& c:[0, \infty) \times \mathbb{R}^{d} \times \Lambda \rightarrow \mathbb{R} \\
& f:[0, \infty) \times \mathbb{R}^{d} \times \Lambda \rightarrow \mathbb{R}
\end{aligned}
$$

be continuous functions such that 
- (Continuity.) $b, c$ and $f$ have the following continuity. For all $T>0, M \geq 1$, there exists $L_{3}(T, M)$ such that for all $t, s \in[0, T],\|x\|,\|y\| \leq M$ and $\alpha, \gamma \in \Lambda$,

- (Locally Lipschitz.)

$$
\|\psi(t, x, \alpha)-\psi(t, y, \gamma)\| \leq L_{3}(T, M)(\|x-y\|+\|\alpha-\gamma\|) .
$$

- (Locally Hölder.)

$$
\|\psi(t, x, \alpha)-\psi(s, x, \alpha)\| \leq L_{3}(T, M)|t-s|^{\beta} .
$$

where $\psi$ represents any of the functions $b, c$ and $f$.

- (Growth.) There exists $c_{0} \geq 0$ such that

$$
c(t, x) \leq c_{0} \quad \text { for all }(t, x, \alpha) \in[0, \infty) \times \mathbb{R}^{d} \times \Lambda .
$$

For all $T>0$ there exist constants $k>0$ and $K_{4}(T)$ such that

$$
\begin{aligned}
\|b(t, x, \alpha)\| & \leq K_{4}(T)(1+\|x\|) \\
|f(t, x, \alpha)| & \leq K_{4}(T)\left(1+\|x\|^{k}\right),
\end{aligned}
$$

for all $0 \leq t \leq T, \alpha \in \Lambda$ and $x \in \mathbb{R}^{d}$

(5) For all $\psi \in H L^{0,1, \beta, \beta_{1}}\left((0, \infty) \times \mathbb{R}^{d}\right), \beta_{1} \in\{\beta, 1\}$, let

$$
A_{\psi}(t, x):=\operatorname{argmax}_{\alpha \in \Lambda}\left\{\mathcal{L}_{1}^{\alpha}[\psi](t, x)+f(t, x, \alpha)\right\} .
$$

Then $A_{\psi} \in H L^{0,0, \beta, \beta_{1}}\left((0, \infty) \times \mathbb{R}^{d}\right)$.

We are ready to prove the main Theorem of this section. The proof is based in Theorem 2.1, the ideas made in Appendix E for Theorem 6.1 of Chapter VI in [21] and some standard arguments for Verification Theorems.

Theorem 3.1. Assume H3. Then there exists a unique classical solution $u \in C\left([0, \infty) \times \mathbb{R}^{d}\right) \cap C_{l o c}^{1,2, \beta}((0, \infty) \times$ $\mathbb{R}^{d}$ ), to equation (33). The solution has the representation

$$
u(t, x)=\sup _{a \in \mathcal{A}}\{u(t, x ; a)\},
$$

where

$$
\begin{aligned}
u(t, x ; a):= & \mathbb{E}_{x}\left[\int_{0}^{t} e^{\int_{0}^{s} c\left(t-r, X(r ; a), a_{r}\right) d r} f\left(t-s, X(s ; a), a_{r}\right) d s\right] \\
& +\mathbb{E}_{x}\left[e^{\int_{0}^{t} c\left(t-r, X(r ; a), a_{r}\right) d r} h(X(t ; a))\right],
\end{aligned}
$$

$\mathcal{A}$ is the set of all predictable processes, $a_{s}$, defined in some probability space $\left(\Omega, \mathcal{F}, \mathbb{P},\left\{\mathcal{F}_{s}\right\}_{s \geq 0}\right)$, such that

$$
\mathbb{P}\left[\text { for all } s \geq 0 ; a_{s} \in \Lambda\right]=1,
$$

and $X(s ; a)$ is the solution to the stochastic differential equation

$$
d X(s ; a)=b\left(t-s, X(s), a_{s}\right) d s+\sigma(t-s, X(s)) d W(s), \quad X(0)=x .
$$

Furthermore, for all $T>0$,

$$
\sup _{0 \leq t \leq T}|u(t, x)| \leq C\left(T, c_{0}, K_{1}(T), K_{2}(T), K_{3}, K_{4}(T), k\right)\left(1+\|x\|^{k}\right), \quad x \in \mathbb{R}^{d},
$$


where $c_{0}, k, K_{i}, i=1, \ldots, 4$ are the constants defined in $\boldsymbol{H 1}, \boldsymbol{H} 2$ and $\boldsymbol{H} 3$.

Remark 3.1. Unlike the linear problem, we restrict the main result for the semilinear part to the case when $\Sigma=\emptyset$. The existence Theorem is proved by approximation with linear equations and requires a Maximum Principle's Theorem in order to prove that the approximating sequence is monotone and hence convergent. In general, is not true that over the set $\partial \Sigma$ the monotonicity is preserved and so we can not construct a convergent sequence by this method. However, as is illustrated in the problem presented in section 4, there exists some cases in which the problem is degenerated in some set $\partial \Sigma \subset \mathbb{R}^{d}$ and repeating the same lines presented in this section we can construct a classical solution to the semilinear problem. In this example, the linearization technique works because the approximating sequence is "constant" over the set $\partial \Sigma$.

The proof is divided in two Theorems: a Verification Theorem and an Existence one.

Theorem 3.2 (Verification Theorem.). Assume H3. Assume also there exists $v \in C\left([0, \infty) \times \mathbb{R}^{d}\right) \cap C_{l o c}^{1,2, \beta}((0, \infty) \times$ $\mathbb{R}^{d}$ ), solution to equation (33) such that for all $T>0$

$$
\sup _{0 \leq t \leq T}|v(t, x)| \leq C(T)\left(1+\|x\|^{\mu}\right), \quad x \in \mathbb{R}^{d},
$$

for some $\mu>0$. Let $a \in \mathcal{A}$, then

$$
u(t, x ; a) \leq v(t, x)=u\left(t, x ; a^{*}\right)
$$

where $a_{s}^{*}:=A_{v}(s, X(s)) \in \mathcal{A}$. In particular, the solution is unique and

$$
v(t, x)=\sup _{a \in \mathcal{A}}\{u(t, x ; a)\}
$$

Proof. Let $\mathcal{L}_{2}$ and $\mathcal{L}_{1}^{\alpha}$ be the differential operators

$$
\mathcal{L}_{2}[u]:=-u_{t}+\sum_{i j} a_{i j} D_{i j} u
$$

and

$$
\mathcal{L}_{1}^{\alpha}[u]:=\sum_{i} b_{i}(\cdot, \alpha) D_{i} u+c(\cdot, \alpha) u
$$

and denote

$$
f^{\alpha}=f(\cdot, \alpha) .
$$

Let $a \in \mathcal{A}$ be any admissible process and denote by $X(s)=X(s ; a)$ the solution to equation $(34)$. Since $v \in C^{1,2}$, applying Ito's rule we get for $s \leq t$

$$
\begin{aligned}
e^{\int_{0}^{s} c\left(t-r, X(r), a_{r}\right) d r} & v(t-s, X(s))=v(t, x) \\
+ & \int_{0}^{s} e^{\int_{0}^{r} c\left(t-y, X(y), a_{y}\right) d y}\left(\mathcal{L}_{2}+\mathcal{L}_{1}^{a_{s}}\right)[v](t-r, X(r)) d r \\
+ & \int_{0}^{s} e^{\int_{0}^{r} c\left(t-y, X(y), a_{y}\right) d y} D v(t-r, X(r)) \sigma(t-r, X(r)) d W(r) .
\end{aligned}
$$

Since $e^{\int_{0}^{r} c d y} D v(\cdot) \sigma(\cdot)$ is locally bounded, we conclude that

$$
\left\{e^{\int_{0}^{s} c\left(t-r, X(r), a_{r}\right) d r} v(t-s, X(s))-\int_{0}^{s} e^{\int_{0}^{r} c\left(t-y, X(y), a_{y}\right) d y}\left(\mathcal{L}_{2}+\mathcal{L}_{1}^{a_{s}}\right)[v](t-r, X(r)) d r\right\}_{0 \leq s \leq t}
$$


is a local martingale. Let $\left\{\tau_{n}\right\}_{n \geq 1}$ be a sequence of localization times for the local martingale. Hence, using equation (33) we get

$$
\begin{aligned}
v(t, x)= & \mathbb{E}_{x}\left[e^{\int_{0}^{s \wedge \tau_{n}} c\left(t-r, X(r), a_{r}\right) d r} v\left((t-\cdot, X)\left(s \wedge \tau_{n}\right)\right)\right] \\
& -\mathbb{E}_{x}\left[\int_{0}^{s \wedge \tau_{n}} e^{\int_{0}^{r} c\left(t-y, X(y), a_{y}\right) d y}\left(\mathcal{L}_{2}+\mathcal{L}_{1}^{a_{s}}\right)[v](t-r, X(r)) d r\right] \\
\geq & \mathbb{E}_{x}\left[e^{\int_{0}^{s \wedge \tau_{n}} c\left(t-r, X(r), a_{r}\right) d r} v\left((t-\cdot, X)\left(s \wedge \tau_{n}\right)\right)\right] \\
& +\mathbb{E}_{x}\left[\int_{0}^{s \wedge \tau_{n}} e^{\int_{0}^{r} c\left(t-y, X(y), a_{y}\right) d y} f\left(t-r, X(r), a_{r}\right) d r\right] .
\end{aligned}
$$

For all $n \in \mathbb{N}$ and $s \leq t$, using (35) we get

$$
\left|e^{\int_{0}^{s \wedge \tau_{n}} c\left(t-r, X(r), a_{r}\right) d r} v\left((t-\cdot, X)\left(s \wedge \tau_{n}\right)\right)\right| \leq e^{c_{0} t} C(t)\left(1+\sup _{r \in[0, t]}\left\{\|X(r)\|^{\mu}\right\}\right),
$$

and

$$
\begin{aligned}
& \int_{0}^{s \wedge \tau_{n}} e^{\int_{0}^{r} c\left(t-y, X(y), a_{y}\right) d y} f\left(t-r, X(r), a_{r}\right) d r \\
& \leq \int_{0}^{s \wedge \tau_{n}} e^{r c_{0}} K_{4}(t)(1+\|X(s)\|)^{k} d s \\
& \leq t e^{t c_{0}} K_{4}(t) 2^{k}\left(1+\sup _{r \in[0, t]}\left\{\|X(r)\|^{k}\right\}\right) .
\end{aligned}
$$

Hence by the Dominated Convergence Theorem letting $n \rightarrow \infty$ and $s \uparrow t$

$$
\begin{aligned}
v(t, x) \geq & \mathbb{E}_{x}\left[\int_{0}^{t} e^{\int_{0}^{s} c\left(t-r, X(r), a_{r}\right) d r} f\left(t-s, X(s), a_{r}\right) d s\right] \\
& +\mathbb{E}_{x}\left[e^{\int_{0}^{t} c\left(t-r, X(r), a_{r}\right) d r} h(X(t))\right] .
\end{aligned}
$$

And so $u(t, x ; a) \leq v(t, x)$.

Since $v \in C_{\text {loc }}^{1,2, \beta}\left((0, \infty) \times \mathbb{R}^{d}\right)$, it follows from $\mathbf{H 3}$ that $A_{v}$ is locally Hölder continuous in $t$ and locally Lipschitz continuous in $x$ and so equation (34) admits a strong solution with $a_{s}^{*}:=A_{v}(s, X(s))$. This implies that the strategy $a_{s}^{*} \in \mathcal{A}$. Repeating the same arguments made with the arbitrary process we get that for $a_{s}^{*}$

$$
u\left(t, x ; a^{*}\right)=v(t, x)
$$

and the proof is complete.

Next we present the Existence Theorem.

Theorem 3.3 (Existence Theorem.). Assume H3. Then there exists a unique classical solution $u \in C([0, \infty) \times$ $\left.\mathbb{R}^{d}\right) \cap C_{\text {loc }}^{1,2, \beta}\left((0, \infty) \times \mathbb{R}^{d}\right)$, to equation (33). Furthermore, for all $T>0$,

$$
\sup _{0 \leq t \leq T}|u(t, x)| \leq C\left(T, c_{0}, K_{1}, K_{2}, K_{3}, K_{4}\right)\left(1+\|x\|^{k}\right), \quad x \in \mathbb{R}^{d},
$$

where $c_{0}, k, K_{i}, i=1, \ldots, 4$ are the constants defined in $\boldsymbol{H} \mathbf{1}, \boldsymbol{H} 2$ and $\boldsymbol{H} 3$. 
Proof. The proof is divided in three main steps: First we construct a candidate solution to equation (33) by approximation with linear parabolic equations. Second we prove that this function is a weak solution to equation (33) and finally we prove that it is a classical solution.

Let $\mathcal{L}_{2}, \mathcal{L}_{1}^{\alpha}$ and $f^{\alpha}$ be defined as in equations (36), (37) and (38), respectively.

Let $\alpha_{0} \in \Lambda$ and $u^{(0)}$ be the solution given by Theorem 2.1 to

$$
\begin{aligned}
\mathcal{L}_{2}\left[u^{(0)}\right]+\mathcal{L}_{1}^{\alpha_{0}}\left[u^{(0)}\right]+f^{\alpha_{0}} & =0, \quad(0, \infty) \times \mathbb{R}^{d} \\
u^{(0)}(0, x) & =h(x), \quad x \in \mathbb{R}^{d} .
\end{aligned}
$$

For $n \geq 1$, let

and $u^{(n)}$ be the solution to

$$
A^{(n-1)}:=\operatorname{argmax}_{\alpha \in \Lambda}\left\{\mathcal{L}_{1}^{\alpha}\left[u^{(n-1)}\right]+f^{\alpha}\right\}
$$

$$
\begin{aligned}
& \mathcal{L}_{2}\left[u^{(n)}\right]+\mathcal{L}_{1}^{A^{(n-1)}}\left[u^{(n)}\right]+f^{A^{(n-1)}}=0, \quad(0, \infty) \times \mathbb{R}^{d} \\
& u^{(n)}(0, x)=h(x), \quad x \in \mathbb{R}^{d} .
\end{aligned}
$$

If $u^{(n-1)} \in C^{1,2, \beta}$ then $D u^{(n-1)} \in C^{0,1, \beta}$ and so $u^{(n-1)} \in H L^{0,1, \beta}$. Hence by hypothesis, $A^{(n-1)} \in H L^{0,0, \beta}$. This and $\mathbf{H 3}$ implies that the coefficients of equation (40) satisfies the hypotheses of Theorem 2.1 and so the sequence $\left\{u^{(n)}\right\}$ is well defined and each $u^{(n)} \in C^{1,2, \beta}$.

Next, we prove that $u^{(n)} \leq u^{(n+1)}$ for all $n \in \mathbb{N}$. Because

$$
A^{(n)} \in \operatorname{argmax}\left\{\mathcal{L}_{1}^{\alpha}\left[u^{(n)}\right]+f^{\alpha}\right\}
$$

we have that

$$
\begin{aligned}
0 & =\mathcal{L}_{2}\left[u^{(n)}\right]+\mathcal{L}_{1}^{A^{(n-1)}}\left[u^{(n)}\right]+f^{A^{(n-1)}} \\
& \leq \mathcal{L}_{2}\left[u^{(n)}\right]+\mathcal{L}_{1}^{A^{(n)}}\left[u^{(n)}\right]+f^{A^{(n)}}
\end{aligned}
$$

Substracting this to equation (40) for $n+1$ we get

$$
0 \geq \mathcal{L}_{2}\left[u^{(n+1)}-u^{(n)}\right]+\mathcal{L}_{1}^{A^{(n)}}\left[u^{(n+1)}-u^{(n)}\right]
$$

in $(0, \infty) \times \mathbb{R}^{d}$. Thanks to the Maximum Principle Theorem 1.2 in [14] we prove that

$$
u^{(n)} \leq u^{(n+1)} .
$$

Since $K_{4}$ does not depend on $\alpha$, then using estimate (8) in Theorem 2.1 we get that for all $n \in \mathbb{N}$

$$
\sup _{0 \leq t \leq T}\left\{\left|u^{(n)}(t, x)\right|\right\} \leq C(T)\left(1+\|x\|^{k}\right), \quad x \in \mathbb{R}^{d}
$$

where the constant $C(T)$ is independent of $n$. Then the sequence $\left\{u^{(n)}(t, x)\right\}$ is bounded from above and increasing. For each $(t, x) \in[0, \infty) \times \mathbb{R}^{d}$, let

$$
u^{*}(t, x):=\lim _{n \rightarrow \infty} u^{(n)}(t, x)
$$

and let $R$ be a bounded, open, connected subset of $(0, \infty) \times \mathbb{R}^{d}$ with smooth boundary. Since all the coefficients of equation (40) are locally bounded, with bound independent of $n$, it follows from Theorem 7.22 in [33] that 
for all $p \geq 1$

$$
\left\|u^{(n)}\right\|_{2, p, R} \leq M_{1} .
$$

where $M_{1}$ does not depend on $n$. The Sobolev space $W^{2, p}(R)$ is embedded in the Hölder space $C^{0,1, \lambda}(R)$, for $0<\lambda \leq 1-\frac{d}{p}$ (see Theorem 4.12 in [1]). Hence for $p$ large enough we can take $\lambda=\beta$ and due the embedding, there exists $M_{2}$ such that for all $n \in \mathbb{N}$

$$
\left|u^{(n)}\right|_{R}^{1, \beta} \leq M_{2} .
$$

So we get that on $R, D u^{(n)}$ converges uniformly to $D u^{*}$, and $u_{t}^{(n)}$ and $D^{2} u^{(n)}$ converge weakly in $L^{p}(R)$ to $u_{t}^{*}$ and $D^{2} u^{*}$ respectively. For $(t, x) \in R$, let

$$
A^{*}(t, x):=\operatorname{argmax}_{\alpha \in \Lambda}\left\{\mathcal{L}_{2}^{\alpha}\left[u^{*}\right](t, x)+f^{\alpha}(t, x)\right\} .
$$

Since $u^{*}$ and $D u^{*}$ are Hölder continuous then by hypotheses, $A^{*} \in C^{0,0, \beta}(R)$. We have the following inequalities

$$
\begin{aligned}
\mathcal{L}_{2}\left[u^{*}\right]+\mathcal{L}_{1}^{A^{*}}\left[u^{*}\right]+f^{A^{*}} & \geq \mathcal{L}_{2}\left[u^{*}\right]+\mathcal{L}_{1}^{A^{(n)}}\left[u^{*}\right]+f^{A^{(n)}} \\
& =\mathcal{L}_{2}\left[u^{*}-u^{(n)}\right]+\mathcal{L}_{1}^{A^{(n)}}\left[u^{*}-u^{(n)}\right] .
\end{aligned}
$$

The righthand side converges weakly to 0 , this implies that that $u^{*}$ satisfies weakly

$$
\mathcal{L}_{2}\left[u^{*}\right]+\mathcal{L}_{1}^{A^{*}}\left[u^{*}\right]+f^{A^{*}} \geq 0 .
$$

On the other side,

$$
\begin{aligned}
\mathcal{L}_{2}\left[u^{(n)}\right]+\mathcal{L}_{1}^{A^{*}}\left[u^{(n)}\right]+f^{A^{*}} & \leq \mathcal{L}_{2}\left[u^{(n)}\right]+\mathcal{L}_{1}^{A^{(n)}}\left[u^{(n)}\right]+f^{A^{(n)}} \\
& =\mathcal{L}_{2}\left[u^{(n)}-u^{(n+1)}\right]+\mathcal{L}_{1}^{A^{(n)}}\left[u^{(n)}-u^{(n+1)}\right] .
\end{aligned}
$$

Again the righthand side converges weakly to 0 in $R$ and so we prove that

$$
\mathcal{L}_{2}\left[u^{*}\right]+\mathcal{L}_{1}^{A^{*}}\left[u^{*}\right]+f^{A^{*}} \leq 0 .
$$

Combining equations (44) and (45) we prove that $u^{*}$ satisfies weakly in $R$

$$
\mathcal{L}_{2}\left[u^{*}\right]+\mathcal{L}_{1}^{A^{*}}\left[u^{*}\right]+f^{A^{*}}=0
$$

It follows from Theorem 4.9 in [33] and the Hölder continuity of $\mathcal{L}_{1}^{A^{*}}\left[u^{*}\right]+f^{A^{*}}$ that

$$
\left|u^{*}\right|_{R}^{2, \beta} \leq C\left(\left\|u^{*}\right\|_{R}+\left|\mathcal{L}_{1}^{A^{*}}\left[u^{*}\right]+f^{A^{*}}\right|_{R}^{\beta}\right),
$$

where $C$ depends on the Hölder norm of the coefficients of $\mathcal{L}_{2}$ and the local ellipticity in $R$. So $u^{*} \in C^{1,2, \beta}(R)$. Since $R$ is arbitrary, then $u^{*} \in C_{\text {loc }}^{1,2, \beta}\left((0, \infty) \times \mathbb{R}^{d}\right)$ and satisfies (33). The initial condition is fulfilled since $u^{(n)}(0, x)=h(x)$ and $h$ is locally Hölder.

Finally, estimate (42) proves that (39) is true and the proof is complete.

\section{Optimal consumption model.}

In this section we present a stochastic optimal consumption model. We consider the wealth of an individual which is dynamically allocated in two investment instruments: a non-risk bonus and a risky asset, both depending on a external factor. The investment strategy is fixed. Our problem is to maximize a logarithmic utility function over all admissible consumption strategies. This kind of problems have been studied in [9], [19] 
and [20] where the authors consider a HARA utility function and the optimization is made over the investment and consumption strategies. One of the main techniques to deal with this probabilistic problem, is to find a differential equation, called a HJB equation, whose solution is the optimal utility function. For this purpose, two main results are needed: a Verification Theorem and an Existence Theorem. The Verification Theorem states that if a solution to the HJB equation exists, then it has to be the optimal utility function and so the solution is unique. Generally, the HJB equations are nonlinear and so the existence of a solution is not trivial. This problem shows situations in which the hypotheses of the present paper appear naturally.

\subsection{The model.}

Let $\left(\Omega, \mathcal{F}, \mathbb{P},\left\{\mathcal{F}_{s}\right\}_{s \geq 0}\right)$ be a complete filtered probability space and let $\left\{W_{1}(s), W_{2}(s)\right\}_{s \geq 0}$ be a two dimensional Brownian motion defined in it. We consider an incomplete market with an external factor

$$
Y(s)=y+\int_{0}^{s} g(Y(r)) d r+\beta\left(\rho W_{1}(s)+\epsilon W_{2}(s)\right),
$$

with $0 \leq \rho \leq 1, \epsilon=\sqrt{1-\rho^{2}}$, and investment instruments

$$
\begin{aligned}
d Z_{0}(s) & =Z_{0}(s) r(Y(s)) d s \\
d Z(s) & =Z(s) \mu(Y(s)) d s+Z(s) \nu(Y(s)) d W_{1}(s) .
\end{aligned}
$$

We assume that $g, \mu, \nu, r: \mathbb{R} \rightarrow \mathbb{R}$ are locally Lipschitz with $\mu, \nu$ and $r$ bounded, $\nu$ strictly positive and $g$ with at most linear growth.

Let $\mathcal{A}$ denote the set of all admissible investment strategies. We select them over all predictable process $A_{s}$ with respect to $\mathcal{F}_{s}$, such that

$$
\mathbb{P}\left[\text { for all } s \geq 0 ; A_{s} \in[0,1]\right]=1
$$

that is, $A_{s}$ denotes the proportion of the wealth consumed at time $s$.

Let $m: \mathbb{R}^{2} \rightarrow \mathbb{R}$ be a bounded, strictly positive, locally Lipschitz continuous function that represents the proportion of the wealth invested in the risky asset.

For $A \in \mathcal{A}$, the wealth process $X(s)$ has the following dynamic

$$
\begin{aligned}
d X(s)= & -A_{s} X(s) d s+X(s)(1-m(X(s), Y(s))) \frac{d Z_{0}(s)}{Z_{0}(s)} \\
& +X(s) m(X(s), Y(s)) \frac{d Z(s)}{Z(s)} \\
= & X(s)\left[-A_{s}+r(Y(s))(1-m(X(s), Y(s)))+\mu(Y(s)) m(X(s), Y(s))\right] d s \\
& +X(s) m(X(s), Y(s)) \nu(Y(s)) d W_{1}(s)
\end{aligned}
$$

with $X(0)=x>0$. This process is strictly positive and has the following representation

$$
X(t)=x \exp \left\{\int_{0}^{t}\left(r(1-m)+\mu m-A_{s}-\frac{1}{2} m^{2} \nu^{2}\right) d s+\int_{0}^{t} m \nu d W_{1}(s)\right\} .
$$

\subsection{The value function and the HJB equation.}

The objective is to maximize the expected consumption utility

$$
V(t, x, y ; A):=\mathbb{E}_{x, y}\left[\int_{0}^{t} \ln \left(A_{s} X(s)+1\right) d s\right]
$$


in a finite horizon, over the set of admissible strategies. Let $V$ be the value function

$$
V(t, x, y):=\sup _{A \in \mathcal{A}}\{V(t, x, y ; A)\} .
$$

To study the regularity of $V$ and the existence of an optimal consumption strategy we consider the following HJB equation

$$
\begin{aligned}
-u_{t} & +\frac{1}{2} x^{2} m(x, y)^{2} \nu(y)^{2} u_{x x}+\beta \rho x m(x, y) \nu(y) u_{x y}+\frac{1}{2} \beta^{2} u_{y y} \\
& +x[r(y)(1-m(x, y))+\mu(y) m(x, y)] u_{x}+g(y) u_{y} \\
& +\sup _{\alpha \in[0,1]}\left\{-\alpha x u_{x}+\ln (\alpha x+1)\right\}=0 \quad(t, x, y) \in(0, \infty) \times(0, \infty) \times \mathbb{R}, \\
& u(0, x, y)=0 \quad(x, y) \in(0, \infty) \times \mathbb{R}^{d} .
\end{aligned}
$$

For simplicity, we omit the $(t, x, y)$ variables in the functions' notation. For any $(x, z) \in(0, \infty) \times \mathbb{R}^{d}$, the function $-x z \alpha+\ln (x \alpha+1)$ is strictly concave in $\alpha$ and has a unique maximum. Then for $\alpha \in[0,1]$ the supremum is attained at

Equation (50) is written as

$$
\psi(x, z):= \begin{cases}0, & \text { if } \frac{1-z}{x z} \leq 0, \\ \frac{1-z}{x z}, & \text { if } 0<\frac{1-z}{x z}<1, \\ 1, & \text { if } 1 \leq \frac{1-z}{x z} .\end{cases}
$$

$$
\begin{aligned}
-u_{t}+\frac{1}{2} x^{2} m^{2} \nu^{2} u_{x x}+\beta \rho x m \nu u_{x y}+\frac{1}{2} \beta^{2} u_{y y} & \\
+x\left[-\psi\left(x, u_{x}\right)+r(1-m)+\mu m\right] u_{x}+g u_{y} & \\
+\ln \left(\psi\left(x, u_{x}\right) x+1\right)=0 & (t, x, y) \in(0, \infty) \times(0, \infty) \times \mathbb{R}, \\
u(0, x, y)=0 & (x, y) \in(0, \infty) \times \mathbb{R} .
\end{aligned}
$$

The coefficients of this equation do not fulfil the ellipticity condition at $x=0$. However, thanks to the kind of degeneracy, we will be able to prove the existence of a classical solution to equation (52).

\subsection{Verification Theorem.}

In this section we propose and prove a Verification Theorem. This Theorem asserts that, in case of existing a classical solution to (52), it has to be the value function and hence is unique. Also, this Theorem proves the existence of an optimal consumption strategy.

Theorem 4.1 (Verification Theorem.). Let $g, \mu, \nu, r: \mathbb{R} \rightarrow \mathbb{R}$ be locally Lipschitz functions such that $\mu, \nu$ and $r$ are bounded, $\nu$ is strictly positive and $g$ has a linear growth. Let $m: \mathbb{R}^{2} \rightarrow \mathbb{R}$ be a bounded, strictly positive and locally Lipschitz function. Assume also there exists $v \in C([0, \infty) \times[0, \infty) \times \mathbb{R}) \cap C_{l o c}^{1,2, \beta}\left((0, \infty) \times(0, \infty) \times \mathbb{R}^{d}\right)$ for some $\beta \in(0,1)$, solution to equation (52) such that for all $T>0$

$$
\sup _{0 \leq t \leq T}|v(t, x, y)| \leq C(T)(1+\|(x, y)\|), \quad(x, y) \in[0, \infty) \times \mathbb{R}^{d} .
$$

Let $A \in \mathcal{A}$ be any admissible strategy, then

$$
V(t, x, y ; A) \leq v(t, x, y)=V\left(t, x, y ; A^{*}\right),
$$

where $A_{s}^{*}=\psi\left(X(s), v_{x}(t-s, X(s), Y(s))\right)$. In particular, the solution is unique and $v(t, x, y)=V(t, x, y)$.

Proof. The proof is similar as the one of Theorem 3.2 observing that the process $X(s ; A)$ is strictly positive and hence we can repeat our analysis restricted to the set $(0, \infty) \times(0, \infty) \times \mathbb{R}$. 


\subsection{Existence of a classical solution to equation (52).}

In this section we prove the existence of a solution to equation (52) with the properties required by the Verification Theorem.

Theorem 4.2. Assume the hypotheses on the functions $r, \mu, \nu, g$ and $m$ made in the Verification Theorem. Then, there exists a unique solution $u \in C\left([0, \infty) \times[0, \infty) \times \mathbb{R}^{d}\right) \cap C_{\text {loc }}^{1,2, \beta}\left((0, \infty) \times(0, \infty) \times \mathbb{R}^{d}\right)$ for $\beta \in(0,1)$, to equation (52) such that for all $T>0$

$$
\sup _{0 \leq t \leq T}|u(t, x, y)| \leq C(T)(1+\|(x, y)\|), \quad(x, y) \in[0, \infty) \times \mathbb{R}^{d} .
$$

Proof. Let $\mathcal{D}_{2}$ and $\mathcal{D}_{1}^{\gamma}$ be the differential operators

$$
\mathcal{D}_{2}[u]:=-u_{t}+\frac{1}{2} x^{2} m^{2} \nu^{2} u_{x x}+\beta \rho x m \nu u_{x y}+\frac{1}{2} \beta^{2} u_{y y}
$$

and

$$
\mathcal{D}_{1}^{\gamma}[u]:=x[r(1-m)+\mu m-\gamma] u_{x}+g u_{y} .
$$

We cannot apply Theorem 3.3 because of the degeneracy of the differential operator $\mathcal{D}_{2}$ at $x=0$, however we can proceed as in its proof.

Equation (52) can be written as

$$
\begin{aligned}
& \mathcal{D}_{2}[u]+\mathcal{D}_{1}^{\psi\left(x, u_{x}\right)}[u]+\ln \left(\psi\left(x, u_{x}\right) x+1\right)=0 \quad \text { in }(0, \infty) \times(0, \infty) \times \mathbb{R} \\
& u(0, x, y)=0 \quad \text { in }(0, \infty) \times \mathbb{R} .
\end{aligned}
$$

Let $u^{(0)}$ be the solution to equation

$$
\begin{aligned}
& \mathcal{D}_{2}\left[u^{(0)}\right]+\mathcal{D}_{1}^{1 / 2}\left[u^{(0)}\right]+\ln \left(\frac{1}{2} x+1\right)=0 \quad \text { in }(0, \infty) \times(0, \infty) \times \mathbb{R} \\
& u^{(0)}(0, x, y)=0 \quad \text { in }[0, \infty) \times \mathbb{R} \text {. }
\end{aligned}
$$

For $n=1,2, \ldots$ let $u^{(n)}$ be the solution to

$$
\begin{aligned}
& \mathcal{D}_{2}\left[u^{(n)}\right]+\mathcal{D}_{1}^{\psi\left(x, u_{x}^{(n-1)}\right)}\left[u^{(n)}\right]+\ln \left(\psi\left(x, u_{x}^{(n-1)}\right) x+1\right)=0 \quad \text { in }(0, \infty) \times(0, \infty) \times \mathbb{R} \\
& u^{(n)}(0, x, y)=0 \quad \text { in }[0, \infty) \times \mathbb{R} \text {. }
\end{aligned}
$$

If $u^{(n-1)} \in C_{\mathrm{loc}}^{1,2, \beta}$ then $u_{x}^{(n-1)} \in C_{\mathrm{loc}}^{0,1, \beta}$ and so is locally Hölder in $t$ and locally Lipschitz in $(x, y)$. Since the function

$$
H(x)= \begin{cases}0, & \text { if } x \leq 0 \\ x, & \text { if } 0<x<1 \\ 1, & \text { if } 1 \leq x\end{cases}
$$

is Lipschitz, then $\psi\left(x, u_{x}^{(n-1)}\right)=H\left(\frac{1-u_{x}^{(n-1)}}{x u_{x}^{(n-1)}}\right)$ is locally Hölder in $t$ and locally Lipschitz in $(x, y)$ whenever $u^{(n-1)}$ is a classical solution.

If we take $\Sigma=(-\infty, 0] \times \mathbb{R}$, thanks to Theorem 2.1 we have that for all $n \in \mathbb{N}$, $u^{(n)}$ is a classical solution to equation (55). To prove that the sequence $\left\{u^{(n)}\right\}$ is increasing we observe that for all $n \in \mathbb{N}, u^{(n)}(t, 0, y) \equiv 0$. So applying the Maximum Principle's Theorem 1.2 in $[14]$ with $\Omega=[0, \infty) \times \mathbb{R}$ we have that

$$
u^{(n)} \leq u^{(n+1)} \quad \text { for all } n \in \mathbb{N} .
$$


We repeat the argument in the proof of Theorem 3.3 to show that $u^{*}:=\lim _{n \rightarrow \infty} u^{(n)}$ is a classical solution to equation (52) and the proof is complete.

\section{Auxiliary Results.}

We need the following results

Theorem 5.1. Let $\left\{\left(t_{n}, x_{n}\right)\right\}_{n \in \mathbb{N}} \subset[0, \infty) \times \mathbb{R}^{d}$ be a sequence such that $\left(t_{n}, x_{n}\right) \underset{n \rightarrow \infty}{\longrightarrow}(t, x)$. Denote by $X_{n}$ and $X$ the solutions of the following equations

$$
d X_{n}(s)=b\left(t_{n}-s, X_{n}(s)\right) d s+\sigma\left(t_{n}-s, X_{n}(s)\right) d W(s), \quad X_{n}(0)=x_{n},
$$

and

$$
d X(s)=b(t-s, X(s)) d s+\sigma(t-s, X(s)) d W(s), \quad X(0)=x .
$$

Then for all $T>0$

$$
\left\|X_{n}-X\right\|_{T} \underset{n \rightarrow \infty}{\stackrel{\mathbb{P}}{\longrightarrow}} 0 .
$$

Proof. This Theorem is consequence of Theorem 1.5 in Chapter V of [32].

The following theorem is Theorem 9 of Chapter 3 in [24]. Let $0 \leq T_{0}<T_{1}$ and let $A \subset \mathbb{R}^{d}$ be a bounded open set with $C^{2}$ boundary. Since $\sigma, b, c$ and $f$ are locally Lipschitz, then they are locally Hölder of any order $\beta \in(0,1)$

Theorem 5.2. Assume $H 1$ and H2. Consider the following Cauchy problem

$$
\begin{gathered}
-u_{t}(t, x)+\mathcal{L}[u](t, x)+c(t, x) u(t, x)=-f(t, x) \quad(t, x) \in\left[T_{0}, T_{1}\right] \times A, \\
u\left(T_{0}, x\right)=g\left(T_{0}, x\right) \quad \text { for } x \in A, \\
u(t, x)=g(t, x) \quad \text { for }(t, x) \in\left(T_{0}, T_{1}\right] \times \partial A .
\end{gathered}
$$

If $g$ is continuous then there exists a classical solution $w \in C\left(\left[T_{0}, T_{1}\right) \times \bar{A}\right) \cap C^{1,2, \beta}\left(\left(T_{0}, T_{1}\right) \times A\right)$ of equation $(56)$.

Remark 5.1. Let $w$ be the solution of equation (56) and define $z$ as $w(t, x)=e^{c_{0} t} z(t, x)$ in $\left[T_{0}, T_{1}\right] \times A$. Then $z$ fulfils equation (56) with $c^{\prime}=c-c_{0}$ and $f^{\prime}(t, x)=e^{c_{0} t} f(t, x)$. And so the hypotheses of Theorem 9 of Chapter 3 in [24] are satisfied.

\section{Acknowledgements.}

I would like to thank specially Begoña Fernández for her valuable comments which helped in developing the results and improving the presentation of this paper. My thanks also go to Daniel Hernández, Ana Meda, Luz de Teresa, Ekaterina Todorova and the participants of the Seminar in Finance and Stochastic Control, UNAMCIMAT for all the discussions on the subject which have been very fruitful and stimulating. The assistance by Nelson Muriel in the presentation of the paper is gratefully acknowledged. Finally, our most sincere gratitude to the reviewers for their very appropriate and precise remarks. The suggested improvements in precision and corrections substantially improved the quality of the present paper. This work was partially supported by grant PAPIIT-DGAPA-UNAM IN103660, IN117109 and CONACYT 180312, México. 


\section{REFERENCES}

[1] Robert A. Adams and John J. F. Fournier. Sobolev spaces, volume 140 of Pure and Applied Mathematics (Amsterdam). Elsevier/Academic Press, Amsterdam, second edition, 2003.

[2] D. G. Aronson and P. Besala. Parabolic equations with unbounded coefficients. J. Differential Equations, 3:1-14, 1967.

[3] Richard F. Bass. Diffusions and elliptic operators. Probability and its Applications (New York). Springer-Verlag, New York, 1998.

[4] M. Bertoldi and S. Fornaro. Gradient estimates in parabolic problems with unbounded coefficients. Studia Math., 165(3):221$254,2004$.

[5] Marcello Bertoldi, Simona Fornaro, and Luca Lorenzi. Gradient estimates for parabolic problems with unbounded coefficients in non convex unbounded domains. Forum Math., 19(4):603-632, 2007.

[6] Marcello Bertoldi and Luca Lorenzi. Estimates of the derivatives for parabolic operators with unbounded coefficients. Trans. Amer. Math. Soc., 357(7):2627-2664 (electronic), 2005.

[7] P. Besala. On the existence of a fundamental solution for a parabolic differential equation with unbounded coefficients. Ann. Polon. Math., 29(4):403-409, 1975. Collection of articles dedicated to the memory of Tadeusz Ważewski, IV.

[8] W. Bodanko. Sur le problème de Cauchy et les problèmes de Fourier pour les équations paraboliques dans un domaine non borné. Ann. Polon. Math., 18:79-94, 1966.

[9] Netzahualcóyotl Castañeda-Leyva and Daniel Hernández-Hernández. Optimal consumption-investment problems in incomplete markets with stochastic coefficients. SIAM J. Control Optim., 44(4):1322-1344 (electronic), 2005.

[10] Sandra Cerrai. Elliptic and parabolic equations in $\mathbf{R}^{n}$ with coefficients having polynomial growth. Comm. Partial Differential Equations, 21(1-2):281-317, 1996.

[11] Sandra Cerrai. Some results for second order elliptic operators having unbounded coefficients. Differential Integral Equations, 11(4):561-588, 1998.

[12] Sandra Cerrai. Analytic semigroups and degenerate elliptic operators with unbounded coefficients: a probabilistic approach. J. Differential Equations, 166(1):151-174, 2000.

[13] Sandra Cerrai. Second order PDE's in finite and infinite dimension, volume 1762 of Lecture Notes in Mathematics. SpringerVerlag, Berlin, 2001. A probabilistic approach.

[14] Lu San Chen, Tadashi Kuroda, and Takaŝi Kusano. Some parabolic equations with unbounded coefficients. Funkcial. Ekvac., 16:1-28, 1973.

[15] Giuseppe Da Prato and Beniamin Goldys. Elliptic operators on $\mathbb{R}^{d}$ with unbounded coefficients. J. Differential Equations, 172(2):333-358, 2001.

[16] Giuseppe Da Prato and Alessandra Lunardi. On the Ornstein-Uhlenbeck operator in spaces of continuous functions. J. Funct. Anal., 131(1):94-114, 1995.

[17] Thomas Deck and Susanne Kruse. Parabolic differential equations with unbounded coefficients - a generalization of the parametrix method. Acta Appl. Math., 74(1):71-91, 2002.

[18] Richard Durrett. Stochastic calculus. Probability and Stochastics Series. CRC Press, Boca Raton, FL, 1996. A practical introduction.

[19] Wendell H. Fleming and Daniel Hernández-Hernández. An optimal consumption model with stochastic volatility. Finance Stoch., 7(2):245-262, 2003.

[20] Wendell H. Fleming and Daniel Hernández-Hernández. The tradeoff between consumption and investment in incomplete financial markets. Appl. Math. Optim., 52(2):219-235, 2005.

[21] Wendell H. Fleming and Raymond W. Rishel. Deterministic and stochastic optimal control. Springer-Verlag, Berlin, 1975. Applications of Mathematics, No. 1.

[22] S. Fornaro, G. Metafune, and E. Priola. Gradient estimates for Dirichlet parabolic problems in unbounded domains. J. Differential Equations, 205(2):329-353, 2004.

[23] Mark Freidlin. Functional integration and partial differential equations, volume 109 of Annals of Mathematics Studies. Princeton University Press, Princeton, NJ, 1985.

[24] Avner Friedman. Partial differential equations of parabolic type. Prentice-Hall Inc., Englewood Cliffs, N.J., 1964.

[25] Avner Friedman. Stochastic differential equations and applications. Vol. 1. Academic Press [Harcourt Brace Jovanovich Publishers], New York, 1975. Probability and Mathematical Statistics, Vol. 28.

[26] Avner Friedman. Stochastic differential equations and applications. Vol. 2. Academic Press [Harcourt Brace Jovanovich Publishers], New York, 1976. Probability and Mathematical Statistics, Vol. 28.

[27] Allan Gut. Probability: a graduate course. Springer Texts in Statistics. Springer, New York, 2005.

[28] Daniel Hernández-Hernández and Alexander Schied. Robust utility maximization in a stochastic factor model. Statist. Decisions, 24(1):109-125, 2006.

[29] Daniel Hernández-Hernández and Alexander Schied. A control approach to robust utility maximization with logarithmic utility and time-consistent penalties. Stochastic Process. Appl., 117(8):980-1000, 2007.

[30] Matthias Hieber, Luca Lorenzi, and Abdelaziz Rhandi. Second-order parabolic equations with unbounded coefficients in exterior domains. Differential Integral Equations, 20(11):1253-1284, 2007. 
[31] Ioannis Karatzas and Steven E. Shreve. Brownian motion and stochastic calculus, volume 113 of Graduate Texts in Mathematics. Springer-Verlag, New York, 1988.

[32] N. V. Krylov. Introduction to the theory of diffusion processes, volume 142 of Translations of Mathematical Monographs. American Mathematical Society, Providence, RI, 1995. Translated from the Russian manuscript by Valim Khidekel and Gennady Pasechnik.

[33] Gary M. Lieberman. Second order parabolic differential equations. World Scientific Publishing Co. Inc., River Edge, NJ, 1996.

[34] Luca Lorenzi and Marcello Bertoldi. Analytical methods for Markov semigroups, volume 283 of Pure and Applied Mathematics (Boca Raton). Chapman \& Hall/CRC, Boca Raton, FL, 2007.

[35] Alessandra Lunardi. Schauder theorems for linear elliptic and parabolic problems with unbounded coefficients in $\mathbf{R}^{n}$. Studia Math., 128(2):171-198, 1998.

[36] Alessandra Lunardi and Vincenzo Vespri. Optimal $L^{\infty}$ and Schauder estimates for elliptic and parabolic operators with unbounded coefficients. In Reaction diffusion systems (Trieste, 1995), volume 194 of Lecture Notes in Pure and Appl. Math., pages 217-239. Dekker, New York, 1998.

[37] Huyên Pham. Smooth solutions to optimal investment models with stochastic volatilities and portfolio constraints. Appl. Math. Optim., 46(1):55-78, 2002.

[38] Philip E. Protter. Stochastic integration and differential equations, volume 21 of Applications of Mathematics (New York). Springer-Verlag, Berlin, second edition, 2004. Stochastic Modelling and Applied Probability.

[39] Alexander Schied. Robust optimal control for a consumption-investment problem. Math. Methods Oper. Res., 67(1):1-20, 2008.

[40] Daniel W. Stroock and S. R. Srinivasa Varadhan. Multidimensional diffusion processes. Classics in Mathematics. SpringerVerlag, Berlin, 2006. Reprint of the 1997 edition.

[41] Constantin Tudor. Procesos estocásticos, volume 2 of Aportaciones Matemáticas: Textos [Mathematical Contributions: Texts]. Sociedad Matemática Mexicana, México, tercera edition, 2002

[42] Thaleia Zariphopoulou. A solution approach to valuation with unhedgeable risks. Finance Stoch., 5(1):61-82, 2001. 